\title{
A Global Optimization Approach for Solving Generalized Nonlinear Multiplicative Programming Problem
}

\author{
Lin-Peng Yang, Pei-Ping Shen, and Yong-Gang Pei \\ College of Mathematics and Information Science, Henan Normal University, Xinxiang 453007, China \\ Correspondence should be addressed to Pei-Ping Shen; shenpp@htu.cn
}

Received 11 February 2014; Accepted 10 March 2014; Published 24 April 2014

Academic Editor: Yisheng Song

Copyright ( 2014 Lin-Peng Yang et al. This is an open access article distributed under the Creative Commons Attribution License, which permits unrestricted use, distribution, and reproduction in any medium, provided the original work is properly cited.

\begin{abstract}
This paper presents a global optimization algorithm for solving globally the generalized nonlinear multiplicative programming (MP) with a nonconvex constraint set. The algorithm uses a branch and bound scheme based on an equivalently reverse convex programming problem. As a result, in the computation procedure the main work is solving a series of linear programs that do not grow in size from iterations to iterations. Further several key strategies are proposed to enhance solution production, and some of them can be used to solve a general reverse convex programming problem. Numerical results show that the computational efficiency is improved obviously by using these strategies.
\end{abstract}

\section{Introduction}

Consider the following generalized nonlinear multiplicative programming problem:

(MP)

$$
\begin{cases}\min & f_{0}(y) \\ \text { s.t. } & f_{j}(y) \leq 1, \quad j=1, \ldots, p, \\ & f_{p+1}(y) \geq 1, \\ & y \in \Omega_{0}=\left\{y \mid 0<y_{i}^{L} \leq y_{i} \leq y_{i}^{U}<\infty,\right. \\ & \quad i=1, \ldots, n\},\end{cases}
$$

where

$$
\begin{array}{r}
f_{j}(y)=\prod_{k=1}^{T_{j}}\left\{\sum_{l=1}^{m_{j}} \beta_{j k_{l}} \prod_{i=1}^{n} y_{i}^{\alpha_{j k_{l i}}}\right\}^{\gamma_{j k}}, \\
j=0,1, \ldots, p+1,
\end{array}
$$

and $\gamma_{j k}, \beta_{j k_{l}}$, and $\alpha_{j k_{l i}}$ are all real numbers with $\gamma_{j k}>0$ and $\beta_{j k_{l}}>0$.

Problem (MP) is worth studying because it frequently appears in many applications, including engineering design [1-6], economics and statistics [7-12], manufacturing [13, 14], chemical equilibrium $[15,16]$, financial optimization [17], plant layout design [18]. On the other hand, many other nonlinear problems, such as quadratic programming $(\mathrm{QP})$, bilinear programming (BLP), linear multiplication programming (LMP), polynomial programming, and generalized geometric programming, fall into the category of (MP).

Problem (MP) usually poses significant theoretical and computational difficulties; that is, it is known to generally possess multiple local optima that are not globally optimal. For example, the problems (LMP), (BLP), and (QP) are multiextremal. Both (LMP) and (MP) are known to be NPhard problems $[19,20]$ and global optimization ones. So, it evoked interest of researchers and practitioners. During the past years, many solution algorithms have been proposed to solve special forms of the problem (MP). The methods can be classified as parameter-based methods [21-23], branchand-bound methods [24-28], outer-approximation methods $[29,30]$, mixed branch-and-bound and outer-approximation method [31], vertex enumeration methods [32, 33], outcomespace cutting plane methods [34], and heuristic methods $[35,36]$.

Up to now, although there has been significant progress in the development of deterministic algorithms for finding global optimal solutions of generalized linear multiplicative programming problem, to our knowledge, little work has been done for globally solving generalized nonlinear multiplicative programming (MP). The purpose of this paper 
is to develop a reliable and effective algorithm for solving problem (MP). In the algorithm, by making use of a variable transformation the original problem (MP) is first equivalently reformulated as a reverse convex programming (RCP). Then, a linear relaxation programming is generated for a lower bound of the optimal value to problem (RCP) in the branchand-bound search by using the exponent and logarithmic functions. Compared with other methods reviewed above, the mathematical model considered in this paper is an important extension for the model given in [24, 26, 37], and the presented linear relaxation technique can be looked upon as an extension application for the one proposed in [24, 26, 37]. Moreover, an upper bound updating strategy is given to provide a better bound than the standard branch-andbound methods (e.g., [24, 26-28, 37]) based on the proposed global solution location rule. Also, the reduction cut given in this paper offers a possibility to cut away a large part of the currently investigated region in which the globally optimal solution of (MP) does not exist. And finally, the numerical results show that the proposed algorithm is feasible and the computational advantages are indicated.

The content of this paper is as follows. In Section 2, we present the problem (RCP) that is equivalent to problem (MP). The four key strategies of the algorithm are detailed in Section 3. A precise algorithm statement and its convergence are given in Section 4. Section 5 reports the numerical results of some sample problems by using the algorithm. Some concluding remarks are given in Section 6.

\section{Equivalent Reformulation}

In this section, we show that any (MP) problem can be transformed into an equivalent reverse convex programming problem with one reverse convex constraint. To see how such a reformulation is possible, some notations will be introduced as follows:

$$
\begin{aligned}
& I=\{1, \ldots, n\}, \\
& J=\{0,1, \ldots, p+1\}, \\
& b_{j k_{l}}=\ln \left(\beta_{j k_{l}}\right), \quad \forall j \in J, k=1, \ldots, T_{j}, l=1, \ldots, m_{j}, \\
& x_{i}=\ln \left(y_{i}\right), \quad i \in I, \\
& L_{i}=\ln \left(y_{i}^{L}\right), \quad U_{i}=\ln \left(y_{i}^{U}\right), \quad \forall i \in I, \\
& \exp (z)=\exp \left(z_{1}\right)+\cdots+\exp \left(z_{m_{j}}\right), \\
& \forall z=\left(z_{1}, \ldots, z_{m_{j}}\right)^{T} \in R^{m_{j}} .
\end{aligned}
$$

Thus, one can convert (MP) into the following equivalent reverse convex programming problem (RCP):

$$
(\mathrm{RCP}) \begin{cases}\min & F_{0}(x) \\ \text { s.t. } & F_{j}(x) \leq 0, \quad j=1, \ldots, p, \\ & F_{p+1}(x) \geq 0, \\ & x \in \bar{\Omega}=\left\{x \mid \bar{L}_{i} \leq x_{i} \leq \bar{U}_{i}, i \in I\right\},\end{cases}
$$

where for each $j \in J$,

$$
F_{j}(x)=\sum_{k=1}^{T_{j}} \gamma_{j k} \ln \left(\sum_{l=1}^{m_{j}} \exp \left(\sum_{i=1}^{n} \alpha_{j k_{l i}} x_{i}+b_{j k_{l}}\right)\right)
$$

are all convex functions. Furthermore, let $A_{j k} \in R^{m_{j} \times n}$ be a matrix with

$$
\begin{aligned}
A_{j k} & \triangleq\left[\begin{array}{cccc}
\alpha_{j k_{11}} & \alpha_{j k_{12}} & \cdots & \alpha_{j k_{1 n}} \\
\alpha_{j k_{21}} & \alpha_{j k_{22}} & \cdots & \alpha_{j k_{2 n}} \\
\cdots & \cdots & \cdots & \cdots \\
\alpha_{j k_{m_{j} 1}} & \alpha_{j k_{m_{j} 2}} & \cdots & \alpha_{j k_{m_{j n}}}
\end{array}\right] \\
& \triangleq\left(\begin{array}{c}
d_{j k_{1}} \\
d_{j k_{2}} \\
\vdots \\
d_{j k_{m_{j}}}
\end{array}\right)
\end{aligned}
$$

and let $b_{j k} \in R^{n}$ be a vector with $b_{j k}=\left(\ln \left(\beta_{j k_{1}}\right), \ln \left(\beta_{j k_{2}}\right)\right.$, $\left.\ldots, \ln \left(\beta_{j k_{m_{j}}}\right)^{T}\right)$. Then, by using (3) $F_{j}(y)$ can be rewritten in the form

$$
\begin{aligned}
F_{j}(x) & =\sum_{k=1}^{T_{j}} \gamma_{j k} \ln \left(\sum_{l=1}^{m_{j}} \exp \left(d_{j k_{l}} x+b_{j k_{l}}\right)\right) \\
& =\sum_{k=1}^{T_{j}} \gamma_{j k} \ln \left(\exp \left(A_{j k} x+b_{j k}\right)\right), \quad j \in J .
\end{aligned}
$$

The key equivalent result for problems (MP) and (RCP) is given by the following theorem.

Theorem 1. If $x^{*}$ is a global optimal solution to problem $(R C P)$, then $y^{*}$ with $y_{i}^{*}=\exp \left(x_{i}^{*}\right), \forall i \in I$, is a global optimal solution for problem (MP). If $y^{*}$ is a global optimal solution for problem (MP), then $x^{*}$ with $x_{i}^{*}=\ln \left(y_{i}^{*}\right), \forall i \in I$, is a global optimal solution for problem $(R C P)$.

Proof. The proof of this theorem follows easily from the definitions of problems (MP) and (RCP); therefore, it is omitted.

\section{Key Strategies of the Algorithm}

From Theorem 1, to globally solve problem (MP), the branchand-bound algorithm to be presented concentrates on globally solving the equivalent problem (RCP). To present the algorithm, we first explain several processes: branching, lower and upper bounding, and reduction cut.

The branching process consists in a successive rectangular partition of the initial box $\bar{\Omega}=[\bar{L}, \bar{U}]$ following in an exhaustive subdivision rule; that is, any infinite nested sequence of partition sets generated through the algorithm shrinks to a singleton. A strategy called the bisection of ratio $\alpha$ will be used in the branching process.

The lower bounding process consists in deriving a linear relaxation programming of problem (RCP) via a two-part 
linearization method. A lower bound for the objective function value $F_{0}(x)$ can be found by solving the linear relaxation programming.

The upper bounding process consists in estimating an upper bound $U^{*}$ for the objective function value $F_{0}(x)$ by adopting a new method in this paper. This method is different from the general method, which is to update the upper bound $U^{*}$ by enclosing all feasible points $x^{k}$ found while computing the lower bounds of the optimum of the primal problem (RCP).

The reduction cut process consists in applying valid cuts (referred to as reduction cuts) to reduce the size of the current partition set $\Omega=[L, U] \subseteq \bar{\Omega}=[\bar{L}, \bar{U}]$. The cuts aim at tightening the box containing the feasible portion currently still of interest.

Next, we will give the four key strategies for forming the corresponding processes, respectively.

3.1. Bisection of Ratio $\alpha$. The algorithm performs a branching process in $R^{n}$ that iteratively subdivides the $n$-dimensional rectangle $\bar{\Omega}$ of problem (RCP) into smaller rectangles that are also of dimension $n$. This process helps the algorithm identify a location in $\bar{\Omega}$ of a point that is a global optimal solution for problem (RCP). At each stage of the process, the subdivision yields a more refined partition [28] of a portion of $\bar{\Omega}$ that is guaranteed to contain a global optimal solution. The initial partition $Q_{0}$ consists simply of $\bar{\Omega}$.

During a typical iteration $k$ of the algorithm, $k \geq 1$, a rectangle $\Omega^{k-1}$ available from iteration $k-1$, is subdivided into two $n$-dimensional rectangles by a process called bisection of ratio $\alpha$, where $\alpha$ is a prechosen parameter that satisfies $0.0<\alpha \leq 0.5$. Let $\Omega^{k-1}=\left\{x \in R^{n} \mid L_{i}^{k-1} \leq x_{i} \leq U_{i}^{k-1}, i \in I\right\}$, where $L_{i}^{k-1}<U_{i}^{k-1}$ for all $i \in I$. The procedure for forming a bisection of ratio $\alpha$ of $\Omega^{k-1}$ into two subrectangles $\bar{\Omega}^{k-1}$ and $\overline{\bar{\Omega}}_{2}^{k-1}$ can be described as follows.

(1) Let

$$
j=\arg \max \left\{U_{i}^{k-1}-L_{i}^{k-1}, i \in I\right\} .
$$

(2) Let $t_{j}$ satisfy

$\min \left\{t_{j}-L_{j}^{k-1}, U_{j}^{k-1}-t_{j}\right\}=\alpha\left(U_{j}^{k-1}-L_{j}^{k-1}\right), \quad 0<\alpha \leq 0.5$.

(3) Let

$$
\begin{gathered}
\bar{\Omega}^{k-1}=\left\{x \in R^{n} \mid L_{i}^{k-1} \leq x_{i} \leq U_{i}^{k-1},\right. \\
\left.i \neq j, L_{j}^{k-1} \leq x_{j} \leq t_{j}, i \in I\right\}, \\
\overline{\bar{\Omega}}^{k-1}=\left\{x \in R^{n} \mid L_{i}^{k-1} \leq x_{i} \leq U_{i}^{k-1},\right. \\
\left.i \neq j, t_{j} \leq x_{j} \leq U_{j}^{k-1}, i \in I\right\} .
\end{gathered}
$$

Clearly, if $\alpha=0.5$, then the bisection of ratio $\alpha$ is the standard bisection rule.
3.2. Linearization Strategy. For each rectangle $\Omega=\left\{x \in R^{n} \mid\right.$ $\left.L_{i} \leq x_{i} \leq U_{i}, i \in I\right\} \subseteq R^{n}$ created by the branching process, the purpose of this linearization strategy is to obtain a lower bound $\operatorname{LB}(\Omega)$ for the optimal value of the problem $(\operatorname{RCP}(\Omega))$.

For each rectangle $\Omega$ created by the branching process, the lower bound $\operatorname{LB}(\Omega)$ is found by solving a single linear relaxation programming $(\operatorname{LRP}(\Omega))$ of problem $(\operatorname{RCP}(\Omega))$. To derive the $(\operatorname{LRP}(\Omega))$, we adopt two-part linearization method. In the first part, we will derive the lower bounding functions and the upper bounding function of each function $\ln \left(\exp \left(A_{j k} x+b_{j k}\right)\right)$. Then, in the second part, we will derive the linear lower bounding function (LLBF) and linear upper bounding function (LUBF) for each sum term of $\exp \left(d_{j k_{l}} x+\right.$ $\left.b_{j k_{l}}\right)$. All the details of this procedure will be given below.

First-part linearization: it is well known that the function $\ln (Y)$ is a concave function about the single variable $Y \in$ $[\underline{Y}, \bar{Y}]$. Let $L(\ln (Y))$ and $U(\ln (Y))$ denote the $(\mathrm{LLBF})$ and (LUBF) of $\ln (Y)$ over the interval $[\underline{Y}, \bar{Y}]$, respectively. Then, from the concavity of $\ln (Y)$, it follows that

$$
\begin{gathered}
L(\ln (Y))=K_{1}(Y-\underline{Y})+\ln (\underline{Y}) \leq \ln (Y), \\
U(\ln (Y))=K_{1} Y-1-\ln K_{1} \geq \ln (Y),
\end{gathered}
$$

where $K_{1}=(\ln \bar{Y}-\ln \underline{Y}) /(\bar{Y}-\underline{Y})$.

Next, to help to derive the (LLBF) and the (LUBF) of each function $F_{j}(x)$, we need to introduce some notations. For any $x \in \Omega$, let

$$
Y_{j k_{l}}^{\Omega}=d_{j k_{l}} x+b_{j k_{l}}
$$

with $d_{j k_{l}}^{T}=\left(a_{j k_{l i}}, \ldots, a_{j k_{l n}}\right)^{T} \in R^{n}$; then we have $Y_{j k_{l}}^{\Omega} \in\left[\underline{Y}_{j k_{l}}^{\Omega}\right.$, $\left.\bar{Y}_{j k_{l}}^{\Omega}\right]$, where

$$
\begin{aligned}
& \underline{Y}_{j k_{l}}^{\Omega}=\sum_{i=1}^{n} \min \left\{a_{j k_{l i}} L_{i}, a_{j k_{l i}} U_{i}\right\}+b_{j k_{l}}, \\
& \bar{Y}_{j k_{l}}^{\Omega}=\sum_{i=1}^{n} \max \left\{a_{j k_{l i}} L_{i}, a_{j k_{l i}} U_{i}\right\}+b_{j k_{l}} .
\end{aligned}
$$

Moreover, let

$$
\eta_{j k}^{\Omega}=\sum_{l=1}^{m_{j}} \exp \left(Y_{j k_{l}}^{\Omega}\right)=\exp \left(A_{j k} x+b_{j k}\right) ;
$$

this will imply $\eta_{j k}^{\Omega} \in\left[\underline{\eta}_{j k}^{\Omega}, \bar{\eta}_{j k}^{\Omega}\right]$, where $\underline{\eta}_{j k}^{\Omega}=\sum_{l=1}^{m_{j}} \exp \left(\underline{Y}_{j k_{l}}^{\Omega}\right)$ and $\bar{\eta}_{j k}^{\Omega}=\sum_{l=1}^{m_{j}} \exp \left(\bar{Y}_{j k_{l}}^{\Omega}\right)$. Denote

$$
K_{j k}^{\Omega}=\frac{\ln \bar{\eta}_{j k}^{\Omega}-\ln \underline{\eta}_{j k}^{\Omega}}{\bar{\eta}_{j k}^{\Omega}-\underline{\eta}_{j k}^{\Omega}} .
$$


Thus, from (11), it follows that the lower bounding function of $\ln \left(\exp \left(A_{j k} x+b_{j k}\right)\right)$ over $\Omega$, denoted as $L_{j k}^{L}(x)$, has the following form:

$$
\begin{aligned}
L_{j k}^{L}(x) & =K_{j k}^{\Omega}\left(\exp \left(A_{j k} x+b_{j k}\right)-\underline{\eta}_{j k}^{\Omega}\right)+\ln \left(\underline{\eta}_{j k}^{\Omega}\right) \\
& =K_{j k}^{\Omega}\left(\sum_{l=1}^{m_{j}} \exp \left(d_{j k_{l}} x+b_{j k_{l}}\right)-\underline{\eta}_{j k}^{\Omega}\right)+\ln \left(\underline{\eta}_{j k}^{\Omega}\right) .
\end{aligned}
$$

Similarly, from (12) the upper bounding function of $\ln \left(\exp \left(A_{j k} x+b_{j k}\right)\right)$ over $\Omega$, denoted as $L_{j k}^{U}(x)$, is as follows:

$$
\begin{aligned}
L_{j k}^{U}(x) & =K_{j k}^{\Omega} \exp \left(A_{j k} x+b_{j k}\right)-1-\ln \left(K_{j k}^{\Omega}\right) \\
& =K_{j k}^{\Omega} \sum_{l=1}^{m_{j}} \exp \left(d_{j k_{l}} x+b_{j k_{l}}\right)-1-\ln \left(K_{j k}^{\Omega}\right) .
\end{aligned}
$$

Based on the previous results, we have

$$
L_{j k}^{L}(x) \leq \ln \left(\exp \left(A_{j k} x+b_{j k}\right)\right) \leq L_{j k}^{U}(x), \quad \forall x \in \Omega .
$$

Therefore, for each $j \in\{0,1, \ldots, p\}$, the first-part lower bounding function of $F_{j}(x)$ in $(7)$, denoted by $L_{j}(x)$, can be given by

$$
\begin{gathered}
L_{j}(x)=\sum_{k=1}^{T_{j}} \gamma_{j k}\left[K_{j k}^{\Omega}\left(\sum_{l=1}^{m_{j}} \exp \left(d_{j k_{l}} x+b_{j k_{l}}\right)-\underline{\eta}_{j k}^{\Omega}\right)\right. \\
\left.+\ln \left(\underline{\eta}_{j k}^{\Omega}\right)\right],
\end{gathered}
$$

and the first-part upper bounding function of $F_{p+1}(x)$ in (7), denoted by $U_{p+1}(x)$, is as follows:

$$
\begin{gathered}
U_{p+1}(x)=\sum_{k=1}^{T_{p+1}} \gamma_{p+1, k}\left[K_{p+1, k}^{\Omega} \sum_{l=1}^{m_{p+1}} \exp \left(d_{p+1, k_{l}} x+b_{p+1, k_{l}}\right)\right. \\
\left.-1-\ln \left(K_{p+1, k}^{\Omega}\right)\right] .
\end{gathered}
$$

Second-part linearization: with a similar method, we can derive the corresponding (LLBF) and (LUBF) of the function $\exp (Y)$ over the interval $\left[Y^{L}, Y^{U}\right]$ such that

$$
K_{2}\left(1+Y-\ln K_{2}\right) \leq \exp (Y) \leq K_{2}\left(Y-Y^{L}\right)+\exp \left(Y^{L}\right),
$$

where

$$
K_{2}=\frac{\exp \left(Y^{U}\right)-\exp \left(Y^{L}\right)}{Y^{U}-Y^{L}} .
$$

From (22), we can derive the (LLBF) and (LUBF) of $\exp \left(Y_{j k_{l}}\right)$ over $\Omega$ as follows:

$$
\begin{aligned}
\exp \left(Y_{j k_{l}}\right) & =\exp \left(d_{j k_{l}} x+b_{j k_{l}}\right) \\
& \geq B_{j k_{l}}^{\Omega}\left(1-\ln \left(B_{j k_{l}}^{\Omega}\right)+d_{j k_{l}} x+b_{j k_{l}}\right), \\
\exp \left(Y_{j k_{l}}\right) & =\exp \left(d_{j k_{l}} x+b_{j k_{l}}\right) \\
& \leq C_{j k_{l}}^{\Omega}+B_{j k_{l}}^{\Omega}\left(d_{j k_{l}} x+b_{j k_{l}}\right),
\end{aligned}
$$

where

$$
\begin{aligned}
B_{j k_{l}}^{\Omega} & =\frac{\exp \left(\bar{Y}_{j k_{l}}^{\Omega}\right)-\exp \left(\underline{Y}_{j k_{l}}^{\Omega}\right)}{\bar{Y}_{j k_{l}}^{\Omega}-\underline{Y}_{j k_{l}}^{\Omega}}, \\
C_{j k_{l}}^{\Omega} & =\frac{\bar{Y}_{j k_{l}}^{\Omega} \exp \left(\underline{Y}_{j k_{l}}^{\Omega}\right)-\underline{Y}_{j k_{l}}^{\Omega} \exp \left(\bar{Y}_{j k_{l}}^{\Omega}\right)}{\bar{Y}_{j k_{l}}^{\Omega}-\underline{Y}_{j k_{l}}^{\Omega}} .
\end{aligned}
$$

Then, for each $j \in\{0,1, \ldots, p\}$, substitute each term $\exp \left(d_{j k_{l}} x+b_{j k_{l}}\right)$ in (20) by $B_{j k_{l}}^{\Omega}\left(1-\ln \left(B_{j k_{l}}^{\Omega}\right)+d_{j k_{l}} x+b_{j k_{l}}\right)$. We may derive the (LLBF) of $F_{j}(x)$ over $\Omega$, denoted as $\operatorname{LF}_{j}(x)$, being the following form:

$$
\begin{aligned}
& \mathrm{LF}_{j}(x) \\
& =\sum_{k=1}^{T_{j}} \gamma_{j k}\left\{K_{j k}^{\Omega}\left[\sum_{l=1}^{m_{j}} B_{j k_{l}}^{\Omega}\left(1-\ln \left(B_{j k_{l}}^{\Omega}\right)+d_{j k_{l}} x+b_{j k_{l}}\right)\right]\right. \\
& \left.\quad-K_{j k}^{\Omega} \underline{\eta}_{j k}^{\Omega}+\ln \left(\underline{\eta}_{j k}^{\Omega}\right)\right\},
\end{aligned}
$$

and it follows that $\operatorname{LF}_{j}(x) \leq F_{j}(x)$ for all $x \in \Omega, j=$ $0,1, \ldots, p$.

If $j=p+1$, substitute the terms $\exp \left(d_{p+1, k_{l}} x+b_{p+1, k_{l}}\right)$ in (21) by $C_{p+1, k_{l}}^{\Omega}+B_{p+1, k_{l}}^{\Omega}\left(d_{p+1, k_{l}} x+b_{p+1, k_{l}}\right)$. We can get the (LUBF) of $F_{p+1}(x)$ over $\Omega$, denoted as $\operatorname{UF}_{p+1}(x)$, as follows:

$$
\begin{aligned}
& \mathrm{UF}_{p+1}(x) \\
& =\sum_{k=1}^{T_{p+1}} \gamma_{p+1, k} \\
& \quad \times\left[K_{p+1, k}^{\Omega} \sum_{l=1}^{m_{p+1}}\left(C_{p+1, k_{l}}^{\Omega}+B_{p+1, k_{l}}^{\Omega}\left(d_{p+1, k_{l}} x+b_{p+1, k_{l}}\right)\right)\right. \\
& \left.\quad-1-\ln \left(K_{p+1, k}^{\Omega}\right)\right],
\end{aligned}
$$

and it follows that $\mathrm{UF}_{p+1}(x) \geq F_{p+1}(x)$ for all $x \in \Omega$. 
From the above discussion for the two kinds of constraints, respectively, we can construct the corresponding linear relaxation programming $(\operatorname{LRP}(\Omega))$ of problem $(\operatorname{RCP}(\Omega))$ as follows:

$$
(\operatorname{LRP}(\Omega)) \begin{cases}\min & \operatorname{LF}_{0}(x) \\ \text { s.t. } & \operatorname{LF}_{j}(x) \leq 0, \quad j=1, \ldots, p, \\ & \operatorname{UF}_{p+1}(x) \geq 0, \\ & x \in \Omega=\left\{x \mid L_{i} \leq x_{i} \leq U_{i}, i \in I\right\} .\end{cases}
$$

Obviously, after the functions are replaced by the corresponding linear functions, the feasible region of problem $(\mathrm{RCP}(\Omega))$ will be contained in the new feasible region of the $(\operatorname{LRP}(\Omega))$, and we can have the following lemma.

Lemma 2. Assume that $L B(\Omega)$ is the minimum of the problem $(L R P(\Omega))$; then $L B(\Omega)$ provides a lower bound for the optimal value of the problem $(R C P(\Omega))$.

3.3. Global Solution Location and Upper Bound Updating. As is known, in the general branch-and-bound algorithm, to update the upper bound $U^{*}$ of the optimal value for problem (RCP), the usual method is enclosing all feasible points found while computing the lower bounds of the optimum of the primal problem (RCP). In this paper, we will adopt a new method to update the upper bounds, which is different from the usual method. Toward this end, firstly, we will give the global solution location.

Let $S=\left\{x \mid F_{j}(x) \leq 0, j=1, \ldots, p\right\}$ and $G=\{x \mid$ $\left.F_{p+1}(x)<0\right\}$. Since the function $F_{j}(x), j=1, \ldots, p+1$, are all convex, both sets $S$ and $G$ are convex. It is clear that the feasible region of the problem (RCP) lies in the set $S \backslash G$. In the problem (RCP), there are two cases at the global solution denoted by $x^{*}$.

Case 1. We have $F_{p+1}\left(x^{*}\right)>0$.

Case 2. We have $F_{p+1}\left(x^{*}\right)=0$.

In the case 1, the reverse convex constraint at the global solution $x^{*}$ is called a nonactive constraint; this nonactive constraint can vanish in the primal problem, so the problem (RCP) is equivalent to the following problem:

$$
\text { (RCP1) } \begin{cases}\min & F_{0}(x) \\ \text { s.t. } & F_{j}(x) \leq 0, \quad j=1, \ldots, p, \\ & x \in \bar{\Omega}=\left\{x \mid \bar{L}_{i} \leq x_{i} \leq \bar{U}_{i}, i \in I\right\},\end{cases}
$$

which is a convex programming and can be solved by many effective algorithms. Obviously, if the optimal solution $x^{*}$ to the above problem satisfies the constraint $F_{p+1}\left(x^{*}\right) \geq 0$, then it will solve (RCP).

In the case 2, the problem (RCP) is equivalent to the following problem:

$$
(\mathrm{RCP} 2) \begin{cases}\min & F_{0}(x) \\ \text { s.t. } & F_{j}(x) \leq 0, \quad j=1, \ldots, p, \\ & F_{p+1}(x)=0, \\ & x \in \bar{\Omega}=\left\{x \mid \bar{L}_{i} \leq x_{i} \leq \bar{U}_{i}, i \in I\right\} .\end{cases}
$$

In this case, we will always have the following assumption.
Assumption 1. A point $x^{\prime}$ is available such that $x^{\prime} \in S$, $F_{p+1}\left(x^{\prime}\right)<0, F_{0}\left(x^{\prime}\right)<\min \left\{F_{0}(x): x \in S \backslash G\right\}$.

Clearly, if point $x^{\prime}$ does not exist, then we only need to solve the problem (RCP1) to obtain the solution of the primal problem (RCP).

In this paper, we will make our efforts to solve the problem in Case 2. It is expedient to indicate some immediate consequences of the above assumption which will locate the solution of (RCP).

Let $\partial G$ denote the bounding of $G$; that is, $\partial G=\{x \mid$ $\left.F_{p+1}(x)=0\right\}$.

For every $x \in(S \cap \Omega) \backslash G$, we can find the point $\widehat{x}$ where the line segment $\left[x^{\prime} ; x\right]$ meets $\partial G$. Clearly it is as follows: $\widehat{x}=$ $t x+(1-t) x^{\prime}$ with $t \in(0,1]$, which can be determined from the following equation:

$$
F_{p+1}(\widehat{x})=0, \quad \text { that is } F_{p+1}\left(t x+(1-t) x^{\prime}\right)=0 \text {. }
$$

Because of the convexity of $S$, and both the points $x$ and $x^{\prime}$ are in the set $S, \widehat{x}$ is in the set $S$ too.

Lemma 3. For every $x \in S \cap \Omega$ such that $F_{p+1}(x)>0$, one has $F_{0}(\widehat{x})<F_{0}(x)$.

Proof. Since $F_{0}\left(x^{\prime}\right)<0$, and $\widehat{x}=t x+(1-t) x^{\prime}$, from the convexity of $F_{0}(x)$ and Assumption 1, we have

$$
\begin{aligned}
F_{0}(\widehat{x}) & \leq t F_{0}(x)+(1-t) F_{0}\left(x^{\prime}\right) \\
& <t F_{0}(x)+(1-t) F_{0}(x)=F_{0}(x)
\end{aligned}
$$

Corollary 4. Under Assumption 1, if $x^{*}$ is the optimal solution of problem (RCP), it lies on $S \cap \partial G$.

According to the discussion of Lemma 3 and Corollary 4, we know that the optimal solution must lie on the boundary, so once a feasible point $x$ is found, we will firstly compute the point $\widehat{x}$ which lies on $S \cap \partial G$ and satisfying $F_{0}(\widehat{x})<F_{0}(x)$, then the upper bound $U^{*}$ of the optimal value for problem (RCP) is updated as

$$
U^{*}=\min \left\{U^{*}, F_{0}(\widehat{x})\right\} .
$$

Hence, once a better upper bound $U^{*}$ is updated, the number of the deleted nodes will increase, and the unnecessary branching and bounding on some regions where the global solution does not exist will decrease greatly.

3.4. Reduction Cut. In this subsection, we pay our attention to how to form the bound reduction technique to accelerate the convergence of the proposed global optimization algorithm.

Assume that $U^{*}$ is a current known upper bound of the optimal objective value of the problem (RCP). For any $\Omega=$ $\left(\Omega_{i}\right)_{n \times 1} \subseteq \bar{\Omega}$ with $\Omega_{i}=\left[L_{i}, U_{i}\right]$, consider the problem $(\operatorname{LRP}(\Omega))$. For the sake of convenience, let the objective 
function and linear constraint functions of $(\operatorname{LRP}(\Omega))$ be expressed as

$$
\begin{aligned}
& \operatorname{LF}_{0}(x)=\sum_{i=1}^{n} c_{i} x_{i}+D \\
& \operatorname{LF}_{j}(x)=\sum_{i=1}^{n} q_{j i} x_{i}-r_{j}, \quad j=1, \ldots, p, \\
& -\mathrm{UF}_{p+1}=\sum_{i=1}^{n} q_{p+1, i} x_{i}-r_{p+1} .
\end{aligned}
$$

Then, from (26) and (27) it follows that

$$
\begin{aligned}
& \left(c_{1}, \ldots, c_{n}\right)^{T}=\sum_{k=1}^{T_{0}} \gamma_{0 k} K_{0 k}^{\Omega} \sum_{l=1}^{m_{0}} B_{0 k_{l}}^{\Omega} d_{0 k_{l}}, \\
& D=\sum_{k=1}^{T_{0}} \gamma_{0 k}\left\{K_{0 k}^{\Omega}\left[\sum_{l=1}^{m_{0}} B_{0 k_{l}}^{\Omega}\left(1-\ln \left(B_{0 k_{l}}^{\Omega}\right)+b_{0 k_{l}}\right)\right]\right. \\
& \left.-K_{0 k}^{\Omega} \underline{\eta}_{0 k}^{\Omega}+\ln \left(\underline{\eta}_{0 k}^{\Omega}\right)\right\} \\
& r_{j}=\sum_{k=1}^{T_{j}} \gamma_{j k}\left\{-K_{j k}^{\Omega}\left[\sum_{l=1}^{m_{j}} B_{j k_{l}}^{\Omega}\left(1-\ln \left(B_{j k_{l}}^{\Omega}\right)+b_{j k_{l}}\right)\right]\right. \\
& \left.+K_{j k}^{\Omega} \underline{\eta}_{j k}^{\Omega}-\ln \left(\underline{\eta}_{j k}^{\Omega}\right)\right\}, \quad j=1, \ldots, p, \\
& r_{p+1}=\sum_{k=1}^{T_{p+1}} \gamma_{p+1, k}\left[K_{p+1, k}^{\Omega} \sum_{l=1}^{m_{p+1}}\left(C_{p+1, k_{l}}^{\Omega}+B_{p+1, k_{l}}^{\Omega} b_{p+1, k_{l}}\right)\right. \\
& \left.-1-\ln \left(K_{p+1, k}^{\Omega}\right)\right] \\
& \left(q_{j 1}, \ldots, q_{j n}\right)^{T}=\sum_{k=1}^{T_{j}} \gamma_{j k} K_{j k}^{\Omega} \sum_{l=1}^{m_{j}} B_{j k_{l}}^{\Omega} d_{j k_{l}} \\
& j=1, \ldots, p, \\
& \left(q_{p+1,1}, \ldots, q_{p+1, n}\right)^{T}=-\sum_{k=1}^{T_{p+1}} \gamma_{p+1, k} K_{p+1, k}^{\Omega} \\
& \times \sum_{l=1}^{m_{p+1}} B_{p+1, k_{l}}^{\Omega} d_{p+1, k_{l}} .
\end{aligned}
$$
lows:

In the following, some notations are introduced as fol-

$$
\begin{aligned}
& r C=\sum_{i=1}^{n} \min \left\{c_{i} L_{i}, c_{i} U_{i}\right\}, \\
& r L_{j}=\sum_{i=1}^{n} \min \left\{q_{j i} L_{i}, q_{j i} U_{i}\right\},
\end{aligned}
$$

$$
\begin{aligned}
& \rho_{i}=U^{*}-r C-D+\min \left\{c_{i} L_{i}, c_{i} U_{i}\right\}, \\
& \delta_{j i}=r_{j}-r L_{j}+\min \left\{q_{j i} L_{i}, q_{j i} U_{i}\right\},
\end{aligned}
$$

where $j=1, \ldots, p+1, i \in I$.

Based on the optimality and feasibility of the problem (RCP), we can give two theorems below to determine the region in which it is guaranteed that there is no optimal solution; thus a reduction cut technique is formed from these theorems.

Theorem 5. For any subrectangle $\Omega=\left(\Omega_{i}\right)_{n \times 1} \subseteq \bar{\Omega}$ with $\Omega_{i}=$ $\left[L_{i}, U_{i}\right]$, the following statements hold.

(i) If $r C+D>U^{*}$, then there exists no optimal solution of the problem (RCP) over the subrectangle $\Omega$.

(ii) If $r C+D \leq U^{*}$, consider the following two cases: if there exists some $t \in I$ satisfying $c_{t}>0$ and $\rho_{t}<c_{t} U_{t}$, then there is no optimal solution of $(R C P)$ over $\Omega_{a}=$ $\left(\Omega_{a i}\right)_{n \times 1}$; conversely, if $c_{t}<0$ and $\rho_{t}<c_{t} L_{t}$ for some $t$, then there does not exist global optimal solution of $(R C P)$ over $\Omega_{b}=\left(\Omega_{b i}\right)_{n \times 1}$, where

$$
\begin{aligned}
& \Omega_{a i}= \begin{cases}\Omega_{i}, & \text { if } i \neq t, \\
\left(\frac{\rho_{t}}{c_{t}}, U_{t}\right] \bigcap \Omega_{t}, & \text { if } i=t,\end{cases} \\
& \Omega_{b i}= \begin{cases}\Omega_{i}, & \text { if } i \neq t, \\
{\left[L_{t}, \frac{\rho_{t}}{c_{t}}\right) \bigcap \Omega_{t},} & \text { if } i=t .\end{cases}
\end{aligned}
$$

Theorem 6. For any $\Omega=\left(\Omega_{i}\right)_{n \times 1} \subseteq \bar{\Omega}$ with $\Omega_{i}=\left[L_{i}, U_{i}\right]$, if $r L_{j}-r_{j}>0$ for some $j \in\{1, \ldots, p+1\}$, then there exists no optimal solution of problem (RCP) over $\Omega$; otherwise, for each $j \in\{1, \ldots, p+1\}$, consider the following two cases.

(i) If there exists some $t \in I$ and $j \in\{1, \ldots, p+1\}$ satisfying $q_{j t}>0$ and $\delta_{j t}<q_{j t} U_{t}$, then there is not optimal solution of the problem (RCP) over $\Omega_{c}=$ $\left(\Omega_{c i}\right)_{n \times 1}$.

(ii) If $q_{j t}<0$ and $\delta_{j t}<q_{j t} L_{t}$ for some $t \in I$ and some $j \in$ $\{1, \ldots, p+1\}$, then no optimal solution of the problem (RCP) over $\Omega_{d}=\left(\Omega_{d i}\right)_{n \times 1}$ exists, where

$$
\begin{aligned}
& \Omega_{c i}= \begin{cases}\Omega_{i}, & \text { if } i \neq t, \\
\left(\frac{\delta_{j t}}{q_{j t}}, U_{t}\right] \bigcap \Omega_{t}, & \text { if } i=t,\end{cases} \\
& \Omega_{d i}= \begin{cases}\Omega_{i}, \\
{\left[L_{t}, \frac{\delta_{j t}}{q_{j t}}\right) \bigcap \Omega_{t},} & \text { if } i=t .\end{cases}
\end{aligned}
$$

Note that the proof of Theorems 5 and 6 is similar to the one of Theorems 1 and 2 in [26]; therefore it is omitted.

Reduction Cut 
(S1) Optimality Cut. Compute $r C+D$. If $r C+D>U^{*}$, and let $\Omega=\emptyset$; otherwise compute $c_{i}(i=1, \ldots, n)$. If $c_{t}>0$ and $\rho_{t}<c_{t} U_{t}$ for some $t \in I$, then let $U_{t}=\rho_{t} / c_{t}$ and $\Omega=\left(\Omega_{i}\right)_{n \times 1}$ with $\Omega_{i}=\left[L_{i}, U_{i}\right](i=1, \ldots, n)$. If $c_{t}<0$ and $\rho_{t}<c_{t} L_{t}$ for some $t \in I$, then let $L_{t}=\rho_{t} / c_{t}$ and $\Omega=\left(\Omega_{i}\right)_{n \times 1}$ with $\Omega_{i}=\left[L_{i}, U_{i}\right](i=1, \ldots, n)$.

(S2) Feasibility Cut. For any $j=1, \ldots, p$, compute $\operatorname{LF}_{j}(x)_{j}$ and $-\mathrm{UF}_{p+1}(x)$. If $r L_{j}-r_{j}>0$ for some $j \in\{1, \ldots, p+1\}$, then let $\Omega=\emptyset$; otherwise compute $q_{j i}(j=1, \ldots, p+1, i=1, \ldots, n)$. If $q_{j t}>0$ and $\delta_{j t}<q_{j t} U_{t}$ for some $j \in\{1, \ldots, p+1\}$ and $t \in I$, then let $U_{t}=\delta_{j t} / q_{j t}$ and $\Omega=\left(\Omega_{i}\right)_{n \times 1}$ with $\Omega_{i}=\left[L_{i}, U_{i}\right](i=$ $1, \ldots, n)$. If $q_{j t}<0$ and $\delta_{j t}<q_{j t} L_{t}$ for some $j \in\{1, \ldots, p+1\}$ and $t \in I$, then let $L_{t}=\delta_{j t} / q_{j t}$ and $\Omega=\left(\Omega_{i}\right)_{n \times 1}$ with $\Omega_{i}=$ $\left[L_{i}, U_{i}\right](i=1, \ldots, n)$.

This reduction cut provides the possibility to cut away a large part of subrectangle $\Omega$ that is currently investigated by the algorithm procedure.

\section{Algorithm and Its Convergence}

Based upon the results and the algorithmic processes discussed in Section 3, the basic steps of the proposed global optimization algorithm are summarized as follows.

Let $\operatorname{LB}(\Omega)$ be the optimal objective function value of $(\operatorname{LRP}(\Omega))$ and $x(\Omega)$ refers to an element of the corresponding argmin.

\subsection{Algorithm Statement}

Step 1 (initialization).

(i) Solve problem (RCP1) with standard convex programming software to obtain the solution $x^{\prime}$ of problem (RCP1). If $F_{p+1}(x) \geq 0$, stop with $x^{\prime}$ as the global solution of the primal problem (RCP).

(ii) Let the set of all active nodes $Q_{0}=\{\bar{\Omega}\}$, the convergence tolerance $\epsilon>0$, the bisection ratio $\alpha \in(0,0.5]$, the upper bound $U^{*}=\infty$, and the iteration counter $k:=0$.

(iii) Find an optimal solution $x(\bar{\Omega})$ and the optimal value $\operatorname{LB}(\bar{\Omega})$ for problem $(\operatorname{LRP}(\bar{\Omega}))$. If $x(\bar{\Omega}) \in(D \cap \bar{\Omega}) \backslash$ $G$, compute $\widehat{x}(\bar{\Omega})$ as given in Section 3.3; set $U^{*}=$ $F_{0}(\widehat{x}(\bar{\Omega}))$. Set the initial lower bound $\operatorname{LB}(0)=\operatorname{LB}(\bar{\Omega})$.

(iv) If $U^{*}-\mathrm{LB}(0) \leq \epsilon$, then stop; $\widehat{x}(\bar{\Omega})$ is a global $\epsilon$-optimal solutions for problem (RCP). Otherwise, go to Step 2.

Step 2 (updating the upper bound). Select the midpoint $x^{\text {mid }}$ of $\Omega^{k}$; if $x^{\text {mid }}$ is feasible to $\left(\operatorname{RCP}\left(\Omega^{k}\right)\right)$, then compute $\widehat{x}\left(\Omega^{k}\right)$ as given in Section 3.3; update the upper bound $U^{*}=$ $\min \left\{U^{*}, F_{0}\left(\widehat{x}\left(\Omega^{k}\right)\right)\right\}$.

Step 3 (reduction). For the subrectangle $\Omega^{k}$ that is currently investigated, we use the reduction cut described in Section 3 to cut away $\Omega^{k}$ and the remaining part is still denoted as $\Omega^{k}$.

Step 4 (branching). Using the strategy of bisection of ratio $\alpha$ described in Section 3.1 to get two new subrectangles and denote the set of new partition rectangles as $\bar{X}_{k}$. For each $\Omega \in \bar{X}_{k}$, compute the lower bound $f_{j}^{l}:=\min _{x \in \Omega} \operatorname{LF}_{j}(x), j=$ $0,1, \ldots, p$ and $f_{p+1}^{u}:=\max _{x \in \Omega} \mathrm{UF}_{p+1}(x)$. If there exists some $j \in\{0,1, \ldots, p\}$ such that one of the lower bounds $f_{j}^{l}$ satisfies $f_{0}^{l}>U^{*}$ or $f_{j}^{l}>0$ for some $j \in\{1, \ldots, p\}$ or $f_{p+1}^{u}<0$, then the corresponding subrectangle $\Omega$ is eliminated from $\bar{X}_{k}$; that is, $\bar{X}_{k}:=\bar{X}_{k} \backslash \Omega$, and skip to the next element of $\bar{X}_{k}$.

Step 5 (bounding). If $\bar{X}_{k} \neq \emptyset$, solve problem $(\operatorname{LRP}(\Omega))$ to obtain $x(\Omega)$ and $\operatorname{LB}(\Omega)$ for each $\Omega \in \bar{X}_{k}$. If $\operatorname{LB}(\Omega)>U^{*}$, set $\bar{X}_{k}:=\bar{X}_{k} \backslash \Omega$. Otherwise, if $x(\Omega) \in(D \bigcap \Omega) \backslash G$, then compute $\widehat{x}(\Omega)$; update the upper bound $U^{*}=\min \left\{U^{*}, F_{0}(\widehat{x}(\Omega))\right\}$ and update $x^{*}$ such that $F_{0}\left(x^{*}\right)=U^{*}$. The partition set remaining is now $Q_{k}:=\left(Q_{k} \backslash \Omega^{k}\right) \cup \bar{X}_{k}$ and a new lower bound is now $\mathrm{LB}(k):=\min \left\{\mathrm{LB}(\Omega) \mid \Omega \in Q_{k}\right\}$.

Step 6 (convergence checking). Set $Q_{k+1}=Q_{k} \backslash\{\Omega \mid \operatorname{LB}(\Omega) \geq$ $\left.U^{*}-\epsilon, \Omega \in Q_{k}\right\}$. If $Q_{k+1}=\emptyset$, then stop with $U^{*}$ as the optimal value and $x^{*}$ as the optimal solution. Otherwise, select an active node $\Omega^{k+1}$ such that $\Omega^{k+1}=\arg \min \{\operatorname{LB}(\Omega) \mid \Omega \epsilon$ $\left.Q_{k+1}\right\}$ for further consideration. Set $k:=k+1$ and return to Step 2.

Next, we give the global convergence of the above algorithm. If the algorithm does not stop finitely, then the branching rule guarantees all the intervals to an isolated point for the variables.

Theorem 7. For any $\Omega=\left\{x \mid L_{i} \leq x_{i} \leq U_{i}, i \in I\right\} \subset \bar{\Omega}$, let $\mu_{i}=U_{i}-L_{i}, \forall i \in I$; then, for any $x \in \Omega, \forall j \in\{0,1, \ldots, p\}$, one has

$$
F_{j}(x)-L F_{j}(x) \longrightarrow 0, \quad F_{p+1}(x)-U F_{p+1}(x) \longrightarrow 0,
$$

as $\mu_{i} \rightarrow 0$ for each $i \in I$.

Proof. First, let us consider the case $j=0,1, \ldots, p$. Let

$$
\Delta_{j}=F_{j}(x)-\operatorname{LF}_{j}(x)=\Delta_{j}^{1}+\Delta_{j}^{2},
$$

where $\Delta_{j}^{1}=F_{j}(x)-L_{j}(x), \Delta_{j}^{2}=L_{j}(x)-\operatorname{LF}_{j}(x)$.

Obviously, if we want to prove $\Delta_{j} \rightarrow 0$ as $\mu_{i} \rightarrow 0, \forall i \in I$, we only need to prove $\Delta_{j}^{1} \rightarrow 0$ and $\Delta_{j}^{2} \rightarrow 0$, as $\mu_{i} \rightarrow 0, \forall i \epsilon$ I.

From (7), (20), and the definition of $\eta_{j k}^{\Omega}$ in (15), we have

$$
\begin{aligned}
\Delta_{j}^{1}= & \sum_{k=1}^{T_{j}} \gamma_{j k} \ln \left(\exp \left(A_{j k} x+b_{j k}\right)\right) \\
& -\sum_{k=1}^{T_{j}} \gamma_{j k}\left[K_{j k}^{\Omega}\left(\exp \left(A_{j k} x+b_{j k}\right)-\underline{\eta}_{j k}^{\Omega}\right)+\ln \left(\underline{\eta}_{j k}^{\Omega}\right)\right] \\
= & \sum_{k=1}^{T_{j}} \gamma_{j k}\left[\ln \left(\eta_{j k}^{\Omega}\right)-K_{j k}^{\Omega}\left(\eta_{j k}^{\Omega}-\underline{\eta}_{j k}^{\Omega}\right)-\ln \left(\underline{\eta}_{j k}^{\Omega}\right)\right]
\end{aligned}
$$


Since $\Delta_{j}^{1}$ is a concave function about $\eta_{j k}^{\Omega}$ for any $\eta_{j k}^{\Omega} \in$ $\left[\underline{\eta}_{j k}^{\Omega}, \bar{\eta}_{j k}^{\Omega}\right]$, it can attain the maximum at the point $\eta_{j k}^{\Omega}=1 / K_{j k}^{\Omega}$. Let $z_{j k}=\bar{\eta}_{j k}^{\Omega} / \eta_{j k}^{\Omega}$; then by computing we have

$$
\begin{aligned}
\max _{x \in \Omega} \Delta_{j}^{1} & \\
= & \sum_{k=1}^{T_{j}} \gamma_{j k}\left[-\ln K_{j k}^{\Omega}-1+K_{j k}^{\Omega} \underline{\eta}_{j k}^{\Omega}-\ln \underline{\eta}_{j k}^{\Omega}\right] \\
& =\sum_{k=1}^{T_{j}} \gamma_{j k}\left[\ln \frac{z_{j k}-1}{\ln z_{j k}}-1+\frac{\ln z_{j k}}{z_{j k}-1}\right] .
\end{aligned}
$$

By the definition of $z_{j k}$, if $\mu_{i} \rightarrow 0, \forall i \in I$, we have $z_{j k} \rightarrow$ 1 , which implies that $\max _{x \in \Omega} \Delta_{j}^{1} \rightarrow 0$ as $\mu_{i} \rightarrow 0, \forall i \in I$. Therefore, we can obtain that

$$
\Delta_{j}^{1} \longrightarrow 0 \quad \text { as } \mu_{i} \longrightarrow 0, \forall i \in I .
$$

Next, we will prove $\Delta_{j}^{2} \rightarrow 0$, as $\mu_{i} \rightarrow 0, \forall i \in I$. From (20) and (26), we have

$$
\begin{aligned}
& \Delta_{j}^{2}=\sum_{k=1}^{T_{j}} \gamma_{j k}\left[K_{j k}^{\Omega}\left(\sum_{l=1}^{m_{j}} \exp \left(d_{j k_{l}} x+b_{j k_{l}}\right)-\underline{\eta}_{j k}^{\Omega}\right)\right.\left.+\ln \left(\underline{\eta}_{j k}^{\Omega}\right)\right] \\
&- \sum_{k=1}^{T_{j}} \gamma_{j k}\left\{K _ { j k } ^ { \Omega } \left[\sum_{l=1}^{m_{j}}\left(B_{j k_{l}}^{\Omega}\left(1-\ln \left(B_{j k_{l}}^{\Omega}\right)+d_{j k_{l}} x+b_{j k_{l}}\right)\right)\right.\right. \\
&\left.=\sum_{k=1}^{T_{j}} \gamma_{j k}\left\{K_{j k}^{\Omega} \quad-\underline{\eta}_{j k}^{\Omega}\right]+\ln \left(\underline{\eta}_{j k}^{\Omega}\right)\right\} \\
& \times\left[\sum_{l=1}^{m_{j}} \exp \left(d_{j k_{l}} x+b_{j k_{l}}\right)\right. \\
&\left.\left.\quad-\sum_{l=1}^{m_{j}}\left(B_{j k_{l}}^{\Omega}\left(1-\ln \left(B_{j k_{l}}^{\Omega}\right)+d_{j k_{l}} x+b_{j k_{l}}\right)\right)\right]\right\} .
\end{aligned}
$$

Now, let us denote

$$
\begin{gathered}
\Delta_{j k}^{21}=\sum_{l=1}^{m_{j}}\left[\exp \left(d_{j k_{l}} x+b_{j k_{l}}\right)\right. \\
\left.-B_{j k_{l}}^{\Omega}\left(1-\ln \left(B_{j k_{l}}^{\Omega}\right)+d_{j k_{l}} x+b_{j k_{l}}\right)\right] \\
=\sum_{l=1}^{m_{j}}\left[\exp \left(Y_{j k_{l}}^{\Omega}\right)-B_{j k_{l}}^{\Omega}\left(1-\ln \left(B_{j k_{l}}^{\Omega}\right)+Y_{j k_{l}}^{\Omega}\right)\right], \\
\Delta_{j k_{l}}^{22}\left(Y_{j k_{l}}^{\Omega}\right)=\exp \left(Y_{j k_{l}}^{\Omega}\right)-B_{j k_{l}}^{\Omega}\left(1-\ln \left(B_{j k_{l}}^{\Omega}\right)+Y_{j k_{l}}^{\Omega}\right) .
\end{gathered}
$$

Then $\Delta_{j}^{2}$ can be rewritten in the following form:

$$
\Delta_{j}^{2}=\sum_{k=1}^{T_{j}} \gamma_{j k} K_{j k}^{\Omega} \sum_{l=1}^{m_{j}} \Delta_{j k_{l}}^{22} .
$$

Since $\Delta_{j k_{l}}^{22}$ is a convex function about $Y_{j k_{l}}^{\Omega}=d_{j k_{l}} x+b_{j k_{l}}$ over the interval $\left[\underline{Y}_{j k_{1}}^{\Omega}, \bar{Y}_{j k_{l}}^{\Omega}\right]$, it follows that $\Delta_{j k_{1}}^{22}$ can attain the maximum $\max _{x \in \Omega} \Delta_{j k_{l}}^{22}$ at the point $\underline{Y}_{j k_{l}}^{\Omega}$ or $\bar{Y}_{j k_{l}}^{\Omega}$. Let $u_{j k_{l}}=$ $\bar{Y}_{j k_{l}}^{\Omega}-\underline{Y}_{j k_{l}}^{\Omega}$ and $v_{j k_{l}}=\left(\exp \left(u_{j k_{l}}\right)-1\right) / u_{j k_{l}}$, then through computing, we can derive that

$$
\begin{aligned}
\Delta_{j k_{l}}^{22}\left(\underline{Y}_{j k_{l}}^{\Omega}\right)= & \exp \left(\underline{Y}_{j k_{l}}^{\Omega}\right)-B_{j k_{l}}^{\Omega}\left(1-\ln \left(B_{j k_{l}}^{\Omega}\right)+\underline{Y}_{j k_{l}}^{\Omega}\right) \\
= & \exp \left(\underline{Y}_{j k_{l}}^{\Omega}\right)-\frac{\exp \left(\bar{Y}_{j k_{l}}^{\Omega}\right)-\exp \left(\underline{Y}_{j k_{l}}^{\Omega}\right)}{\bar{Y}_{j k_{l}}^{\Omega}-\underline{Y}_{j k_{l}}^{\Omega}} \\
& \times\left(1-\ln \frac{\exp \left(\bar{Y}_{j k_{l}}^{\Omega}\right)-\exp \left(\underline{Y}_{j k_{l}}^{\Omega}\right)}{\bar{Y}_{j k_{l}}^{\Omega}-\underline{Y}_{j k_{l}}^{\Omega}}+\underline{Y}_{j k_{l}}^{\Omega}\right) \\
= & \exp \left(\underline{Y}_{j k_{l}}^{\Omega}\right)\left(1-v_{j k_{l}}\left(1-\ln v_{j k_{l}}\right)\right), \\
\Delta_{j k_{l}}^{22}\left(\bar{Y}_{j k_{l}}^{\Omega}\right)= & \exp \left(\bar{Y}_{j k_{l}}^{\Omega}\right)-B_{j k_{l}}^{\Omega}\left(1-\ln \left(B_{j k_{l}}^{\Omega}\right)+\bar{Y}_{j k_{l}}^{\Omega}\right) \\
= & \exp \left(\bar{Y}_{j k_{l}}^{\Omega}\right)-\frac{\exp \left(\bar{Y}_{j k_{l}}^{\Omega}\right)-\exp \left(\underline{Y}_{j k_{l}}^{\Omega}\right)}{\bar{Y}_{j k_{l}}^{\Omega}-\underline{Y}_{j k_{l}}^{\Omega}} \\
& \times\left(1-\ln \frac{\exp \left(\bar{Y}_{j k_{l}}^{\Omega}\right)-\exp \left(\underline{Y}_{j k_{l}}^{\Omega}\right)}{\bar{Y}_{j k_{l}}^{\Omega}-\underline{Y}_{j k_{l}}^{\Omega}}+\bar{Y}_{j k_{l}}^{\Omega}\right) \\
= & \exp \left(\bar{Y}_{j k_{l}}^{\Omega}\right) \\
& -\exp \left(\underline{Y}_{j k_{l}}^{\Omega}\right) v_{j k_{l}}\left(1-\ln v_{j k_{l}}+\bar{Y}_{j k_{l}}^{\Omega}-\underline{Y}_{j k_{l}}^{\Omega}\right) .
\end{aligned}
$$

Additionally, by the definitions of $\underline{Y}_{j k_{l}}^{\Omega}$ and $\bar{Y}_{j k_{l}}^{\Omega}$, we know that for any $j, k, l, \bar{Y}_{j k_{l}}^{\Omega}-\underline{Y}_{j k_{l}}^{\Omega} \rightarrow 0$ and $\exp \left(\bar{Y}_{j k_{l}}^{\Omega}\right)-\exp \left(\underline{Y}_{j k_{l}}^{\Omega}\right) \rightarrow 0$ as $\mu_{i} \rightarrow 0, \forall i \in I$, and so, we have $v_{j k_{l}}$ go to 1 as each $\mu_{i}$ approaches zero. This implies that $\Delta_{j k_{l}}^{22}\left(\underline{Y}_{j k_{l}}^{\Omega}\right) \rightarrow 0$ and $\Delta_{j k_{l}}^{22}\left(\bar{Y}_{j k_{l}}^{\Omega}\right) \rightarrow 0$ as $\mu_{i} \rightarrow 0, \forall i \in I$; that is, $\max _{x \in \Omega} \Delta_{j k_{l}}^{22} \rightarrow 0$ as $\mu_{i} \rightarrow 0, \forall i \in I$. Hence, according to the above discussion, we can follow that

$$
\Delta_{j}^{2} \longrightarrow 0, \quad \text { as } \mu_{i} \longrightarrow 0, \forall i \in I .
$$

Based on the above discussion, for each $j=0,1, \ldots, p$, from (40) it follows that

$$
\Delta_{j} \longrightarrow 0, \quad \text { as } \mu_{i} \longrightarrow 0, \forall i \in I .
$$

Second, let us consider $j=p+1$. Let

$$
\Delta_{p+1}=\mathrm{UF}_{p+1}(x)-F_{p+1}(x)=\Delta_{p+1}^{1}+\Delta_{p+1}^{2},
$$


where $\Delta_{p+1}^{1}=U_{p+1}(x)-F_{p+1}(x), \Delta_{p+1}^{2}=\mathrm{UF}_{p+1}(x)-U_{p+1}(x)$. By using (7), (21), and the definition of $\eta_{p+1, k}^{\Omega}$ in (15) we have

$$
\begin{aligned}
& \Delta_{p+1}^{1}= U_{p+1}(x)-F_{p+1}(x) \\
&= \sum_{k=1}^{T_{p+1}} \gamma_{p+1, k}\left[K_{p+1, k}^{\Omega} \exp \left(A_{p+1, k} x+b_{p+1, k}\right)\right. \\
&\left.-1-\ln \left(K_{p+1, k}^{\Omega}\right)\right] \\
&-\sum_{k=1}^{T_{p+1}} \gamma_{p+1, k} \ln \left(\exp \left(A_{p+1, k} x+b_{p+1, k}\right)\right) \\
&= \sum_{k=1}^{T_{p+1}} \gamma_{p+1, k}\left[K_{p+1, k}^{\Omega} \sum_{l=1}^{m_{p+1}} \exp \left(d_{p+1, k_{l}} x+b_{p+1, k_{l}}\right)\right. \\
&\left.-\ln \left(\sum_{l=1}^{m_{p+1}} \exp \left(d_{p+1, k_{l}} x+b_{p+1, k_{l}}\right)\right)\right] \\
&= \sum_{k=1}^{T_{p+1}} \gamma_{p+1, k}\left[K_{p+1, k}^{\Omega} \eta_{p+1, k}^{\Omega}-1\right. \\
&\left.-\ln \left(K_{p+1, k}^{\Omega}\right)-\ln \left(\eta_{p+1, k}^{\Omega}\right)\right] .
\end{aligned}
$$

Since $\Delta_{p+1}^{1}$ is a convex function about $\eta_{p+1, k}^{\Omega}$ over the interval $\left[\underline{\eta}_{p+1, k}^{\Omega}, \bar{\eta}_{p+1, k}^{\Omega}\right]$, it can attain the maximum $\max _{x \in \Omega} \Delta_{p+1}^{1}$ at the point $\underline{\eta}_{p+1, k}^{\Omega}$ or $\bar{\eta}_{p+1, k}^{\Omega}$. Let $z_{p+1, k}=\bar{\eta}_{p+1, k}^{\Omega} / \underline{\eta}_{p+1, k}^{\Omega}$; then, by similar discussion as above we can obtain that

$$
\begin{aligned}
& \Delta_{p+1}^{1}\left(\underline{\eta}_{p+1, k}^{\Omega}\right) \\
& =\sum_{k=1}^{T_{p+1}} \gamma_{p+1, k}\left[K_{p+1, k}^{\Omega} \underline{\eta}_{p+1, k}^{\Omega}-1-\ln \left(K_{p+1, k}^{\Omega}\right)-\ln \left(\underline{\eta}_{p+1, k}^{\Omega}\right)\right] \\
& =\sum_{k=1}^{T_{p+1}} \gamma_{p+1, k}\left(\ln \frac{z_{p+1, k}-1}{\ln z_{p+1, k}}-1+\frac{\ln z_{p+1, k}}{z_{p+1, k}-1}\right), \\
& \Delta_{p+1}^{1}\left(\bar{\eta}_{p+1, k}^{\Omega}\right) \\
& =\sum_{k=1}^{T_{p+1}} \gamma_{p+1, k}\left[K_{p+1, k}^{\Omega} \bar{\eta}_{p+1, k}^{\Omega}-1-\ln \left(K_{p+1, k}^{\Omega}\right)-\ln \left(\bar{\eta}_{p+1, k}^{\Omega}\right)\right] \\
& =\sum_{k=1}^{T_{p+1}} \gamma_{p+1, k}\left(\ln \frac{z_{p+1, k}-1}{\ln z_{p+1, k}}\right. \\
& \left.-1+\frac{z_{p+1, k} \ln z_{p+1, k}}{z_{p+1, k}-1}-\ln z_{p+1, k}\right) .
\end{aligned}
$$

Since $z_{p+1, k} \rightarrow 1$ as $\mu_{i} \rightarrow 0, \forall i \in I$, we have that $\Delta_{p+1}^{1}\left(\underline{\eta}_{p+1, k}^{\Omega}\right) \rightarrow 0$ and $\Delta_{p+1}^{1}\left(\bar{\eta}_{p+1, k}^{\Omega}\right) \rightarrow 0$; that is, $\max _{x \in \Omega} \Delta_{p+1}^{1} \rightarrow 0$, as $\mu_{i} \rightarrow 0, \forall i \in I$. Hence, according to the above discussion, we can obtain that

$$
\Delta_{p+1}^{1} \longrightarrow 0, \quad \text { as } \mu_{i} \longrightarrow 0, \forall i \in I .
$$

On the other hand, from (27) and (21) it follows that $\Delta_{p+1}^{2}$

$$
\begin{aligned}
& =\mathrm{UF}_{p+1}(x)-U_{p+1}(x) \\
& =\sum_{k=1}^{T_{p+1}} \gamma_{p+1, k} \\
& \quad \times\left[K_{p+1, k}^{\Omega} \sum_{l=1}^{m_{p+1}}\left(C_{p+1, k_{l}}^{\Omega}+B_{p+1, k_{l}}^{\Omega}\left(d_{p+1, k_{l}} x+b_{p+1, k_{l}}\right)\right)\right. \\
& \left.\quad-1-\ln \left(K_{p+1, k}^{\Omega}\right)\right]
\end{aligned}
$$$$
-\sum_{k=1}^{T_{p+1}} \gamma_{p+1, k}\left[K_{p+1, k}^{\Omega} \sum_{l=1}^{m_{p+1}} \exp \left(d_{p+1, k_{l}} x+b_{p+1, k_{l}}\right)\right.
$$$$
\left.-1-\ln \left(K_{p+1, k}^{\Omega}\right)\right]
$$

$$
\begin{aligned}
& =\sum_{k=1}^{T_{p+1}} \gamma_{p+1, k} K_{p+1, k}^{\Omega} \\
& \quad \times \sum_{l=1}^{m_{p+1}}\left[C_{p+1, k_{l}}^{\Omega}+B_{p+1, k_{l}}^{\Omega}\left(d_{p+1, k_{l}} x+b_{p+1, k_{l}}\right)\right. \\
& \left.\quad-\exp \left(d_{p+1, k_{l}} x+b_{p+1, k_{l}}\right)\right] \\
& =\sum_{k=1}^{T_{p+1}} \gamma_{p+1, k} K_{p+1, k}^{\Omega} \\
& \quad \times \sum_{l=1}^{m_{p+1}}\left[C_{p+1, k_{l}}^{\Omega}+B_{p+1, k_{l}}^{\Omega} Y_{p+1, k_{l}}^{\Omega}-\exp \left(Y_{p+1, k_{l}}^{\Omega}\right)\right] .
\end{aligned}
$$

Now, let us denote

$$
\Delta_{p+1, k_{l}}^{21}=C_{p+1, k_{l}}^{\Omega}+B_{p+1, k_{l}}^{\Omega} Y_{p+1, k_{l}}^{\Omega}-\exp \left(Y_{p+1, k_{l}}^{\Omega}\right) ;
$$

then, $\Delta_{p+1}^{2}$ can be rewritten as follows:

$$
\Delta_{p+1}^{2}=\sum_{k=1}^{T_{p+1}} \gamma_{p+1, k} K_{p+1, k}^{\Omega} \sum_{l=1}^{m_{p+1}} \Delta_{p+1, k_{l}}^{21}
$$

Since $\Delta_{p+1, k_{l}}^{21}$ is a concave function about $Y_{p+1, k_{l}}^{\Omega}$ over the inter$\operatorname{val}\left[\underline{Y}_{p+1, k_{l}}^{\Omega}, \bar{Y}_{p+1, k_{l}}^{\Omega}\right]$, it attains the maximum $\max _{x \in \Omega} \Delta_{p+1, k_{l}}^{21}$ at 
the point $Y_{p+1, k_{l}}^{\Omega}=\ln \left(B_{p+1, k_{l}}^{\Omega}\right)$. Let $u_{p+1, k_{l}}=\bar{Y}_{p+1, k_{l}}^{\Omega}-\underline{Y}_{p+1, k_{l}}^{\Omega}$ and $v_{p+1, k_{l}}=\left(\exp \left(u_{p+1, k_{l}}\right)-1\right) / u_{p+1, k_{l}}$; then by computing we can get

$$
\begin{aligned}
\max _{x \in \Omega} \Delta_{p+1, k_{l}}^{21} & \\
= & C_{p+1, k_{l}}^{\Omega}+B_{p+1, k_{l}}^{\Omega} \ln \left(B_{p+1, k_{l}}^{\Omega}\right)-B_{p+1, k_{l}}^{\Omega} \\
= & -B_{p+1, k_{l}}^{\Omega} \underline{Y}_{p+1, k_{l}}^{\Omega}+\exp \left(\underline{Y}_{p+1, k_{l}}^{\Omega}\right) \\
& +B_{p+1, k_{l}}^{\Omega} \ln \left(B_{p+1, k_{l}}^{\Omega}\right)-B_{p+1, k_{l}}^{\Omega} \\
= & \exp \left(\underline{Y}_{p+1, k_{l}}^{\Omega}\right)\left[v_{p+1, k_{l}} \ln v_{p+1, k_{l}}+1-v_{p+1, k_{l}}\right] .
\end{aligned}
$$

By the definition of $u_{p+1, k_{l}}$ and $v_{p+1, k_{l}}$, we have $u_{p+1, k_{l}} \rightarrow 0$ and $v_{p+1, k_{l}} \rightarrow 1$ as $\mu_{i} \rightarrow 0, \forall i \in I$. This implies that $\max _{x \in \Omega} \Delta_{p+1, k_{l}}^{21} \rightarrow 0$ as $\mu_{i} \rightarrow 0, \forall i \in I$. Therefore, we have

$$
\Delta_{p+1}^{2} \longrightarrow 0, \quad \text { as } \mu_{i} \longrightarrow 0, \forall i \in I .
$$

Thus, by (50) and the above discussion, it is obvious that

$$
\Delta_{p+1} \longrightarrow 0 \quad \text { as } \mu_{i} \longrightarrow 0, \forall i \in I .
$$

In summary, according to the above results, the proof is complete.

Remark 8. From Theorem 7, it follows that $\operatorname{LF}_{j}(x), j=$ $0,1, \ldots, p$ and $\mathrm{UF}_{p+1}(x)$ will approximate the corresponding functions $F_{j}(x), j=0,1, \ldots, p$ and $F_{p+1}(x)$ as $\mu_{i} \rightarrow 0, \forall i \in$ $I$.

Theorem 9. (a) If the algorithm is finite, then upon termination, the incumbent solution being optimal to $(R C P)$ is a global $\epsilon$-optimal solution for problem (RCP).

(b) If the algorithm is infinite, then it will generate an infinite sequence of iterations such that along any infinite branch of the branch and bound tree, any accumulation point of the sequence $\left\{\widehat{x}^{k}\right\}$ will be the global solution of the problem $(R C P)$.

Proof. (a) If the algorithm is finite, then it terminates in some Step $k, k \geq 0$. Without loss of generality, upon termination, the incumbent solution is denoted as $\widehat{x}^{k}$. By the algorithm, it follows that $U^{*}-\mathrm{LB}(k) \leq \epsilon$. From (iv) of Steps 1 and 6, this implies that $F_{0}\left(\widehat{x}^{k}\right)-\mathrm{LB}(k) \leq \epsilon$. Let $v$ denote the optimal value of problem (RCP); then, by Section 3, we know that $\mathrm{LB}(k) \leq$ $v$. Since $\widehat{x}^{k}$ is a feasible solution of problem (RCP), we have $f\left(\widehat{x}^{k}\right) \geq v$. Taken together, this implies that

$$
F_{0}\left(\hat{x}^{k}\right) \geq v \geq \operatorname{LB}(k) \geq F_{0}\left(\hat{x}^{k}\right)-\epsilon .
$$

Therefore, $\widehat{x}^{k}$ is a global $\epsilon$-optimal solution for problem (RCP). And the proof of part (a) is complete.

(b) When the algorithm is infinite, a sufficient condition for a global optimization to be convergent to the global minimum, stated in [28], requires that the bounding operation must be consistent and the selection operation is bound improving.
A bounding operation is called consistent if at every step any unfathomed partition can be further refined, and if any infinitely decreasing sequence of successively refined partition elements satisfies

$$
\lim _{k \rightarrow \infty}\left(\mathrm{UB}_{k}-\mathrm{LB}_{k}\right)=0
$$

where $\mathrm{UB}_{k}$ is a computed upper bound in stage $k$ and $\mathrm{LB}_{k}$ is the best lower bound at iteration $k$ not necessarily occurring inside the same subrectangle with $\mathrm{UB}_{k}$. In the following, we will show that (61) holds.

Since the employed subdivision process is exhaustive. Consequently, from Theorem 7 and the relationship $V(\mathrm{RCP}) \geq V(\mathrm{LRP})$, the formulation (61) holds; this implies that the employed bounding operation is consistent.

A selection operation is called the bound improving if at least one partition element where the actual upper bound is attained is selected for further partition after a finite number of refinements. Clearly, the employed selection operation is the bound improving because the partition element where the actual upper bound is attained is selected for further partition in the immediately following iteration.

In summary, we have shown that the bounding operation is consistent and that selection operation is the bound improving. Therefore, according to Theorem IV.3 in [28], the employed global optimization algorithm is convergent to the global minimum of (RCP).

\section{Numerical Experiments}

To demonstrate the potentiality and feasibility of the proposed global optimization algorithm, our numerical experiment is reported in this section. The algorithm is coded in $\mathrm{C}++$ and each linear programming is solved by the simplex method. The convergence tolerance $\epsilon$ is set to $\epsilon=10^{-5}$ in our experiments.

Example 10 (see [37]). Consider

$$
\begin{array}{ll}
\min & x_{1} \\
\text { s.t. } & x_{1}^{-1} x_{2}^{2}+x_{1}^{-1} x_{3}^{2} \leq 1, \\
& 0.3 x_{2} x_{3} \geq 1, \\
& x \in X=\left\{x \mid 1 \leq x_{1} \leq 100,\right. \\
& \left.1 \leq x_{2} \leq 100,1 \leq x_{3} \leq 100\right\} .
\end{array}
$$

Example 11 (see [37]). Consider

$$
\begin{array}{ll}
\min & x_{1} \\
\text { s.t. } & 3.7 x_{1}^{-1} x_{2}^{0.85}+1.985 x_{1}^{-1} x_{2}+700.3 x_{1}^{-1} x_{3}^{-0.75} \leq 1, \\
& 0.7673 x_{3}^{0.05} x_{4}^{-1} \leq 1, \\
& x_{4}^{-1}+0.05 x_{2} x_{4}^{-1} \geq 1,
\end{array}
$$




$$
\begin{array}{r}
x \in X=\left\{x \mid 0.1 \leq x_{1} \leq 15,0.1 \leq x_{2} \leq 5,\right. \\
\left.380 \leq x_{3} \leq 450,0.1 \leq x_{4} \leq 10\right\} .
\end{array}
$$

Example 12 (see [37]). Consider

$$
\begin{array}{ll}
\min & x_{3}^{0.8} x_{4}^{1.2} \\
\text { s.t. } & x_{1} x_{4}^{-1}+x_{2}^{-1} x_{4}^{-1} \leq 1, \\
& x_{1}^{-2} x_{3}^{-1}+x_{2} x_{3}^{-1} \geq 1, \\
& x \in X=\left\{x \mid 0.1 \leq x_{1} \leq 1,5 \leq x_{2} \leq 10,\right. \\
& \left.8 \leq x_{3} \leq 15,0.01 \leq x_{4} \leq 1\right\} .
\end{array}
$$

Example 13 (see [26]). Consider

$$
\begin{array}{ll}
\min & 5 x_{1}+50000 x_{1}^{-1}+20 x_{2}+72000 x_{2}^{-1}+144000 x_{3}^{-1} \\
\text { s.t. } & 4 x_{1}^{-1}+32 x_{2}^{-1}+120 x_{3}^{-1} \leq 1, \\
& 70 \leq x_{1} \leq 108,83 \leq x_{2} \leq 100, \\
& 200 \leq x_{2} \leq 210 .
\end{array}
$$

Example 14 (see [24]). Consider

$$
\begin{array}{ll}
\min & \left(x_{1}+x_{2}+x_{3}\right)\left(2 x_{1}+x_{2}+x_{3}\right)\left(x_{1}+2 x_{2}+2 x_{3}\right) \\
\text { s.t. } & \left(x_{1}+2 x_{2}+x_{3}\right)^{1.1}\left(2 x_{1}+2 x_{2}+x_{3}\right)^{1.3} \leq 100, \\
& 1 \leq x_{1}, x_{2}, x_{3} \leq 3 .
\end{array}
$$

Example 15 (see [24]). Consider

$$
\begin{array}{ll}
\min & \left(x_{1}+x_{2}+1\right)^{2.5}\left(2 x_{1}+x_{2}+1\right)^{1.1}\left(x_{1}+2 x_{2}+1\right)^{1.9} \\
\text { s.t. } & \left(x_{1}+2 x_{2}+1\right)^{1.1}\left(2 x_{1}+2 x_{2}+2\right)^{1.3} \leq 50, \\
& 1 \leq x_{1} \leq 3,1 \leq x_{2} \leq 3 .
\end{array}
$$

\section{Example 16. Consider}

$$
\begin{array}{cl}
\min & \left(x_{1} x_{2}^{-2}+x_{2}^{0.8} x_{3}^{-1.2}\right)\left(x_{1}^{-1.2} x_{2}^{-2}+x_{3}^{0.8} x_{4}^{-1.2}\right)^{12} \\
\text { s.t. } \quad & \left(5 x_{2}^{3} x_{3}^{0.5}+10 x_{1} x_{3}^{-2}\right)\left(23 x_{2}^{-3} x_{3}^{0.5}+12 x_{1} x_{4}^{-2}\right)^{8} \leq 1, \\
& \left(5 x_{1}^{-1.2} x_{2}^{2}+20 x_{1}^{-1} x_{3}^{5}\right)^{0.8}\left(x_{1} x_{4}^{-1}+x_{2}^{-1} x_{4}^{-1}\right)^{2.4} \leq 1, \\
& \left(8 x_{2} x_{3}^{1.1}+2 x_{2} x_{3}\right)\left(0.2 x_{1}^{-2} x_{3}^{5}+0.6 x_{2} x_{4}^{-1}\right) \geq 1, \\
x \in X=\left\{x \mid 0.1 \leq x_{1} \leq 20,1 \leq x_{2} \leq 50,\right. \\
\left.1 \leq x_{3} \leq 60,0.01 \leq x_{4} \leq 40\right\} .
\end{array}
$$

TABLE 1: $\alpha=0.5$; the reduction cut and new upper bound updating are not adopted.

\begin{tabular}{lcccc}
\hline Example & 3 & 4 & 5 & 6 \\
\hline Iter & 300 & 1596 & 83 & 471 \\
$L_{\max }$ & 73 & 436 & 22 & 54 \\
Time & 0.9105 & 12.2033 & 0.2504 & 2.2001 \\
\hline
\end{tabular}

TABLE 2: $\alpha=0.5$; the reduction cut is not adopted.

\begin{tabular}{lcccc}
\hline Example & 3 & 4 & 5 & 6 \\
\hline Iter & 300 & 1596 & 52 & 457 \\
$L_{\max }$ & 70 & 432 & 6 & 44 \\
Time & 0.8903 & 11.3474 & 0.4003 & 52.4406 \\
\hline
\end{tabular}

TABLE 3: $\alpha=0.5$; the strategies in Section 3 are all adopted.

\begin{tabular}{lcccc}
\hline Example & 3 & 4 & 5 & 6 \\
\hline Iter & 139 & 145 & 68 & 64 \\
$L_{\max }$ & 55 & 73 & 11 & 10 \\
Time & 0.4378 & 0.6898 & 0.3475 & 0.3026 \\
\hline
\end{tabular}

In order to test the effectiveness of several key strategies in Section 3, we select the above four Examples 12-15 by adopting the different strategy in the branch-and-bound search, the corresponding computational results are summarized in Tables $1,2,3$, and 4 , respectively.

In Table 1, by setting $\alpha=0.5$, the test does not adopt the reduction cut (i.e., Step 3 of the algorithm is skipped), and the new method to update upper bounds has not been applied; that is, $U^{*}=\min \left\{U^{*}, F_{0}(\widehat{x}(\Omega)\}\right.$ is replaced by $U^{*}=$ $\min \left\{U^{*}, F_{0}(x(\Omega)\}\right.$ in (iii) of Steps 1 and 5 of the algorithm. In Tables 2-4, the test can be refereed the corresponding of explanation in the header of tables.

In these tables, some notations have been used for column headers, that is, Iter: the number of the algorithm iterations; $L_{\text {max }}$ : the maximal number of the active nodes necessary; and Time: the execution time in seconds.

The computational results show that the proposed algorithm can globally solve the problem (MP) effectively. Furthermore, comparing the numerical results, from Tables 1-4 it is shown that the proposed several strategies, especially in the reduction cut, upper bound updating, and bisection of ratio $\alpha$, are very effective for decreasing the number of the iteration and the maximal number of the active nodes and the running CPU time.

Additionally, in order to test our algorithm further, we give some other computational results, which are generated randomly. In Table 5 below, the convergence tolerance parameters are set as $10^{-6}$, where the average CPU times (denoted by Ave. time), average number of iterations (denoted by Ave. Iter), and average longest node number (denoted by Ave. $L$ ) are obtained by running the algorithm for 10 times. 
TABLE 4: $\alpha=0.35$; the four strategies are all adopted in Section 3.

\begin{tabular}{lccccccc}
\hline Number & Ref. & Iter & $L_{\max }$ & Time & Optimal solution & Optimal value \\
\hline \multirow{2}{*}{1} & Ours & 105 & 38 & 0.2192 & $(6.6667,1.8257,1.8258)$ & 6.6667 \\
& {$[37]$} & 151 & 33 & 0.15 & $(6.6667,1.8257,1.8258)$ & 6.6667 \\
\hline \multirow{2}{*}{} & Ours & 113 & 64 & 0.2279 & $(11.9644,0.8112,442.66,1.0406)$ & 11.9644 \\
& {$[37]$} & 259 & 104 & 0.62 & $(11.9632,0.8158,444.6624,1.0408)$ & 11.9632 \\
\hline 3 & Ours & 5 & 2 & 0.0574 & $(0.1000,10.0000,8.0000,0.2000)$ & 0.7651 \\
& {$[37]$} & 99 & 27 & 0.15 & $(0.1000,10.0000,8.0000,0.2000)$ & 0.7651 \\
\hline 4 & Ours & 8 & 3 & 0 & $(100,83,210.0)$ & 60.0 \\
6 & Ours & 1 & 1 & 0 & $(1.0,1.0,1.0)$ & 9213.184165257 \\
7 & Ours & 1 & 1 & 0 & $(1.0,1.0)$ & 997.661265 \\
\hline
\end{tabular}

TABLE 5: Numerical results for random problems.

\begin{tabular}{ccccccc}
\hline$T$ & $p$ & $m$ & $n$ & Ave. Iter & Ave. $L$ & Ave. time \\
\hline 2 & 3 & 3 & 6 & 2811 & 402 & 2.9228 \\
2 & 3 & 3 & 8 & 12342 & 2832 & 17.225 \\
2 & 5 & 3 & 6 & 3954 & 556 & 6.7927 \\
2 & 3 & 5 & 6 & 5832 & 758 & 10.9702 \\
4 & 3 & 3 & 6 & 6346 & 706 & 14.2749 \\
4 & 5 & 3 & 8 & 38043 & 7695 & 173.6108 \\
4 & 5 & 3 & 6 & 8739 & 1240 & 16.7542 \\
4 & 3 & 5 & 6 & 11969 & 1757 & 36.6518 \\
\hline
\end{tabular}

Example 17. Consider

$$
\begin{aligned}
& \min \quad F_{0}(y)=\prod_{k=1}^{T}\left(\sum_{l=1}^{m} \beta_{0 k_{l}} \prod_{i=1}^{n} y_{i}^{\alpha_{0 k_{l i}}}\right)^{\gamma_{0 k}} \\
& \text { s.t. } \quad F_{j}(y)=\prod_{k=1}^{T}\left(\sum_{l=1}^{m} \beta_{j k_{l}} \prod_{i=1}^{n} y_{i}^{\alpha_{j k_{l i}}}\right)^{\gamma_{j k}} \leq 1, \\
& F_{p+1}(y)=\prod_{k=1}^{T}\left(\sum_{l=1}^{m} \beta_{p+1, k_{l}} \prod_{i=1}^{n} y_{i}^{\alpha_{p+1, k_{l i}}}\right)^{\gamma_{p+1, k}} \geq 1, \\
& y \in \Omega=\left\{y \mid y_{i}^{L} \leq y_{i} \leq y_{i}^{U}, i \in I\right\},
\end{aligned}
$$

where $\gamma_{j k}, \alpha_{j k_{l i}}$, and $\beta_{j k_{l}}$ are generated randomly in the intervals $[1,2],[0,5]$, and $[0.1,10.1]$, respectively. And $y_{i}^{L}$ and $y_{i}^{U}$ are generated randomly in the intervals $[0,1]$ and $[10,11]$.

It is seen from Table 5 that the sizes of $T$ and $n$ are the main factors affecting the performance of the algorithm. This is mainly because the number of terms in the subproblem linear programs $(\operatorname{LRP}(\Omega))$ is proportional to $T$ or $n$. Also, the CPU time increases as $p$ or $m$ increases, but not as sharply as $T$ or $n$.

\section{Concluding Remarks}

A deterministic global optimization algorithm is proposed for solving problem (MP). It successfully reduces a complicated problem (MP) to a simpler reverse convex programming (RCP) problem. Based on the characteristics of the problem (RCP), several global optimization strategies are proposed. The first one is bisection of ratio $\alpha$, which provides a more flexible subdivision rule. The second strategy is the linearization method. By adopting the two-part linearization method, the linear relaxation programming of the problem (RCP) can be obtained, whose minimum will provide the lower bound of the minimum of the problem (RCP). The third strategy is global solution location and upper bound updating. This strategy provides a method to locate the global solutions of the (RCP) and decreases the maximal number of the active nodes and the computational effort required during the algorithm. The final strategy is reduction cut as an accelerating device, which can cut away all part or a large part of the currently investigated feasible region in which the global optimal solution does not exist. A branch and bound algorithm is presented in which the four strategies are adopted successfully. The proposed algorithm is convergent to the global solutions. And the numerical results show that our algorithm is effective and feasible. It is noted that the third strategy can be used for solving the general reverse convex programming problems effectively.

\section{Conflict of Interests}

The authors declare that there is no conflict of interests regarding the publication of this paper.

\section{Acknowledgment}

This research was supported by the National Natural Science Foundation of China $(11171094 ; 11171368)$.

\section{References}

[1] M. Avriel and A. C. Williams, "An extension of geometric programming with applications in engineering optimization," 
Journal of Engineering Mathematics, vol. 5, no. 2, pp. 187-199, 1971.

[2] T. R. Jefferson and C. H. Scott, "Generalized geometric programming applied to problems of optimal control. I. Theory," Journal of Optimization Theory and Applications, vol. 26, no. 1, pp. 117-129, 1978.

[3] K. J. Nand, "Geometric programming based robot control design," Computers and Industrial Engineering, vol. 29, no. 1-4, pp. 631-635, 1995.

[4] K. Das, T. K. Roy, and M. Maiti, "Multi-item inventory model with quantity-dependent inventory costs and demand-dependent unit cost under imprecise objective and restrictions: a geometric programming approach," Production Planning and Control, vol. 11, no. 8, pp. 781-788, 2000.

[5] T. Dwyer, Y. Koren, and K. Marriott, "Drawing directed graphs using quadratic programming," IEEE Transactions on Visualization and Computer Graphics, vol. 12, no. 4, pp. 536-548, 2006.

[6] J. A. M. Petersen and M. Bodson, "Constrained quadratic programming techniques for control allocation," IEEE Transactions on Control Systems Technology, vol. 14, no. 1, pp. 91-98, 2006.

[7] J. C. Choi and D. L. Bricker, "Effectiveness of a geometric programming algorithm for optimization of machining economics models," Computers and Operations Research, vol. 23, no. 10, pp. 957-961, 1996.

[8] H. El Barmi and R. L. Dykstra, "Restricted multinomial maximum likelihood estimation based upon Fenchel duality," Statistics and Probability Letters, vol. 21, no. 2, pp. 121-130, 1994.

[9] D. L. Bricker, K. O. Kortanek, and L. Xu, "Maximum likelihood estimates with order restrictions on probabilities and odds ratios: a geometric programming approach," Journal of Applied Mathematics and Decision Sciences, vol. 1, no. 1, pp. 53-65, 1997.

[10] R. Jagannathan, "A stochastic geometric programming problem with multiplicative recourse," Operations Research Letters, vol. 9, no. 2, pp. 99-104, 1990.

[11] L. L. Abdel-Malek and N. Areeratchakul, "A quadratic programming approach to the multi-product newsvendor problem with side constraints," European Journal of Operational Research, vol. 176, no. 3, pp. 1607-1619, 2007.

[12] H. G. Schwarz, "Economic materials-product chain models: current status, further development and an illustrative example," Ecological Economics, vol. 58, no. 2, pp. 373-392, 2006.

[13] A. I. Sönmez, A. Baykasoǧlu, T. Dereli, and I. H. Filiz, "Dynamic optimization of multipass milling operations via geometric programming," International Journal of Machine Tools and Manufacture, vol. 39, no. 2, pp. 297-320, 1999.

[14] C. H. Scott and T. R. Jefferson, "Allocation of resources in project management," International Journal of Systems Science, vol. 26, no. 2, pp. 413-420, 1995.

[15] C. D. Maranas and C. A. Floudas, "Global optimization in generalized geometric programming," Computers and Chemical Engineering, vol. 21, no. 4, pp. 351-369, 1997.

[16] M. J. Rijckaert and X. M. Martens, "Analysis and optimization of the Williams-Otto process by geometric programming," AIChE Journal, vol. 20, no. 4, pp. 742-750, 1974.

[17] C. D. Maranas, I. P. Androulakis, C. A. Floudas, A. J. Berger, and J. M. Mulvey, "Solving long-term financial planning problems via global optimization," Journal of Economic Dynamics and Control, vol. 21, no. 8-9, pp. 1405-1425, 1997.

[18] I. Quesada and I. E. Grossmann, "Alternative bounding approximations for the global optimization of various engineering design problems," in Global Optimization in Engineering Design,
Nonconvex Optimization and Its Applications, I. E. Grossmann, Ed., vol. 9, pp. 309-331, Kluwer Academic Publishers, Norwell, Mass, USA, 1996.

[19] H. Konno, T. Kuno, and Y. Yajima, "Parametric simplex algorithms for a class of NP-Complete problems whose average number of steps is polynomial," Computational Optimization and Applications, vol. 1, no. 2, pp. 227-239, 1992.

[20] T. Matsui, "NP-hardness of linear multiplicative programming and related problems," Journal of Global Optimization, vol. 9, no. 2, pp. 113-119, 1996.

[21] H. Konno, T. Kuno, and Y. Yajima, "Global minimization of a generalized convex multiplicative function," Journal of Global Optimization, vol. 4, no. 1, pp. 47-62, 1994.

[22] H. Konno, Y. Yajima, and T. Matsui, "Parametric simplex algorithms for solving a special class of nonconvex minimization problems," Journal of Global Optimization, vol. 1, no. 1, pp. 6581, 1991.

[23] N. V. Thoai, "A global optimization approach for solving the convex multiplicative programming problem," Journal of Global Optimization, vol. 1, no. 4, pp. 341-357, 1991.

[24] P. P. Shen and H. W. Jiao, "Linearization method for a class of multiplicative programming with exponent," Applied Mathematics and Computation, vol. 183, no. 1, pp. 328-336, 2006.

[25] H. S. Ryoo and N. V. Sahinidis, "Global optimization of multiplicative programs," Journal of Global Optimization, vol. 26, no. 4, pp. 387-418, 2003.

[26] P. P. Shen and H. W. Jiao, "A new rectangle branch-and-pruning approach for generalized geometric programming," Applied Mathematics and Computation, vol. 183, no. 2, pp. 1027-1038, 2006.

[27] C. F. Wang and S. Y. Liu, "A new linearization method for generalized linear multiplicative programming," Computers and Operations Research, vol. 38, no. 7, pp. 1008-1013, 2011.

[28] T. Kuno, "A finite branch-and-bound algorithm for linear multiplicative programming," Computational Optimization and Applications, vol. 20, no. 2, pp. 119-135, 2001.

[29] Y. Gao, C. Xu, and Y. Yang, "An outcome-space finite algorithm for solving linear multiplicative programming," Applied Mathematics and Computation, vol. 179, no. 2, pp. 494-505, 2006.

[30] T. Kuno, Y. Yajima, and H. Konno, "An outer approximation method for minimizing the product of several convex functions on a convex set," Journal of Global Optimization, vol. 3, no. 3, pp. 325-335, 1993.

[31] H. P. Benson, "An outcomes pace branch and bound-outer approximation algorithm for convex multiplicative programming," Journal of Global Optimization, vol. 15, no. 4, pp. 315-342, 1999.

[32] K. P. Bennett, "Global tree optimization: anon-greedy decision tree algorithm," Computing Sciences and Statistics, vol. 26, pp. 156-160, 1994.

[33] P. M. Pardalos, "Polynomial time algorithms for some classes of constrained quadratic problems," Optimization, vol. 21, no. 6, pp. 843-853, 1990.

[34] H. P. Benson and G. M. Boger, "Outcome-space cutting-plane algorithm for linear multiplicative programming," Journal of Optimization Theory and Applications, vol. 104, no. 2, pp. 301322, 2000.

[35] H. P. Benson and G. M. Boger, "Multiplicative programming problems: analysis and efficient point search heuristic," Journal of Optimization Theory and Applications, vol. 94, no. 2, pp. 487510, 1997. 
[36] X. J. Liu, T. Umegaki, and Y. Yamamoto, "Heuristic methods for linear multiplicative programming," Journal of Global Optimization, vol. 15, no. 4, pp. 433-447, 1999.

[37] Y. Wang and Y. Lan, "Global optimization for special reverse convex programming," Computers and Mathematics with Applications, vol. 55, no. 6, pp. 1154-1163, 2008. 


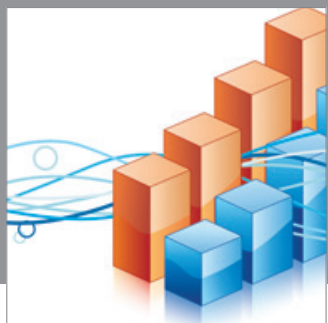

Advances in

Operations Research

mansans

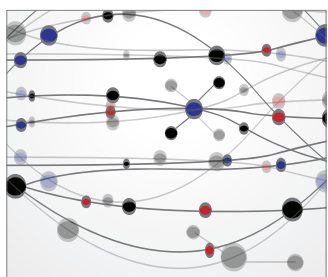

The Scientific World Journal
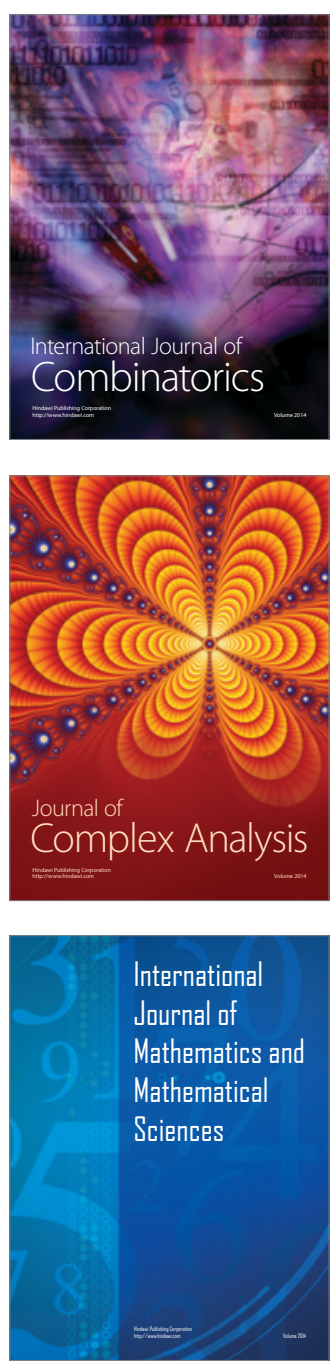
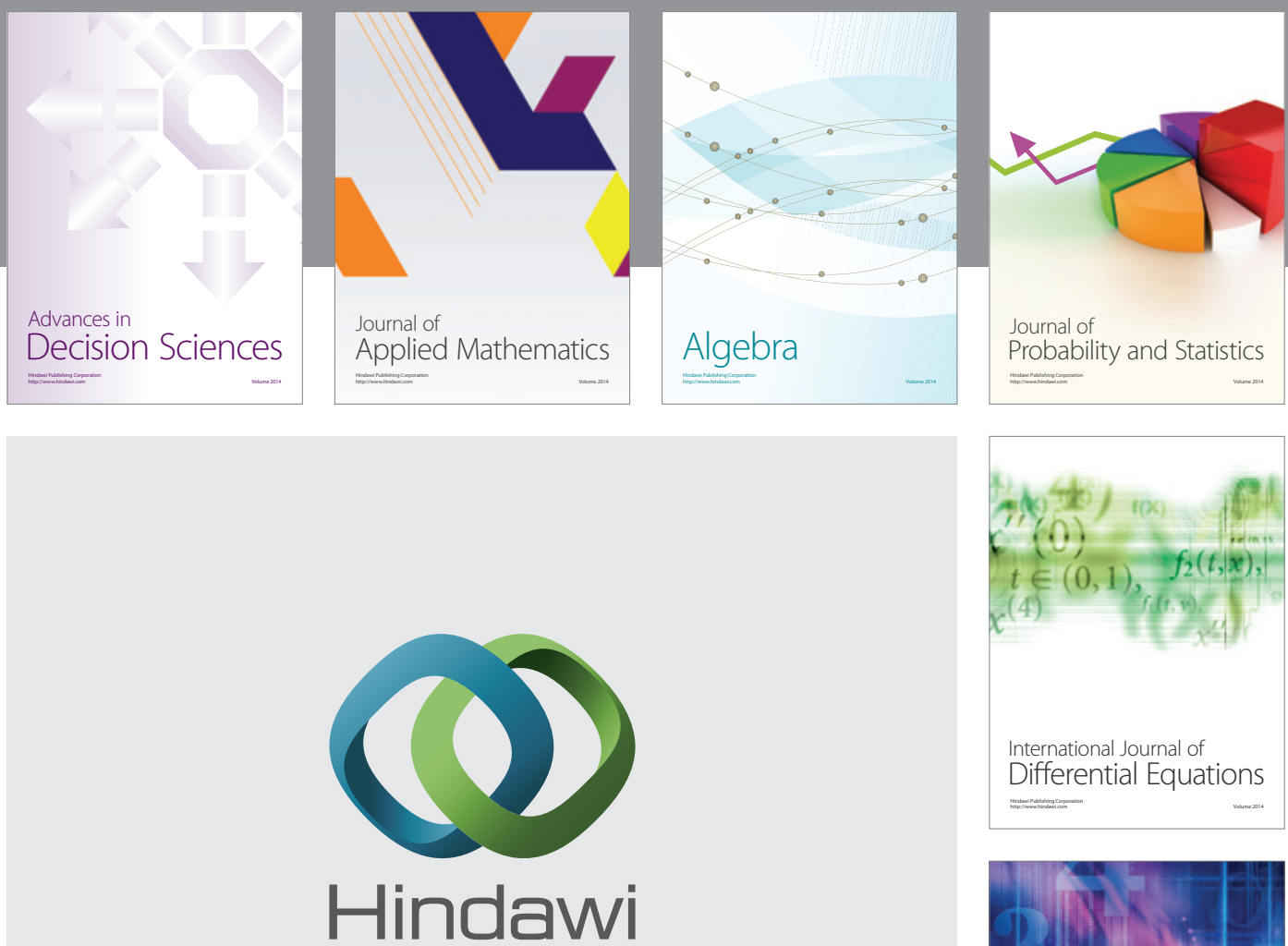

Submit your manuscripts at http://www.hindawi.com
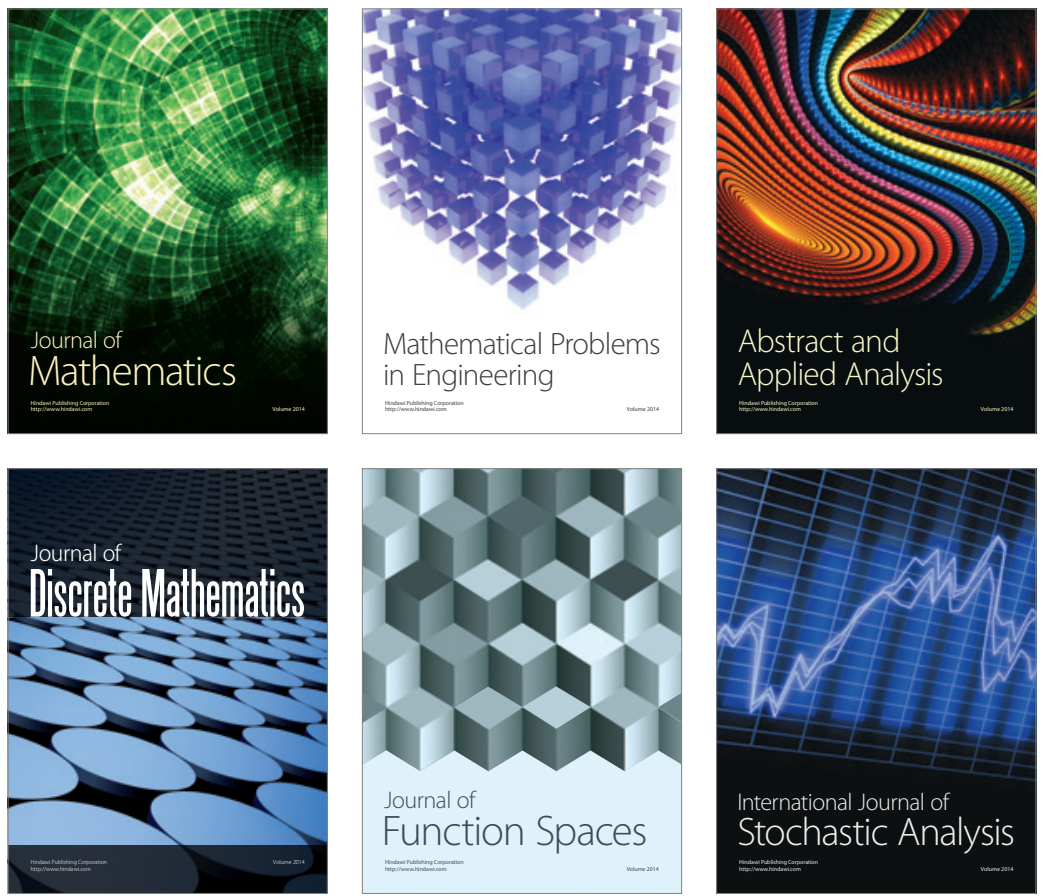

Journal of

Function Spaces

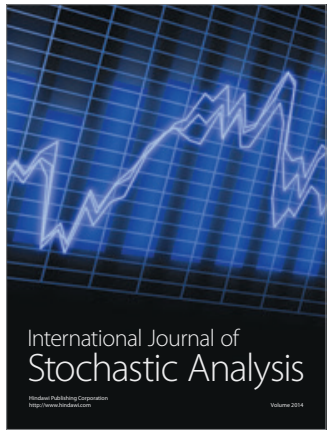

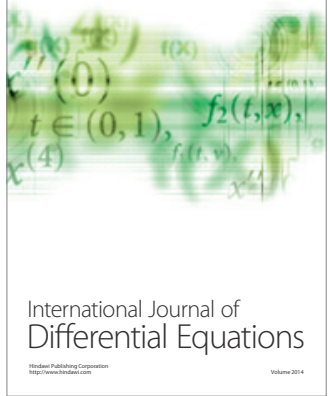
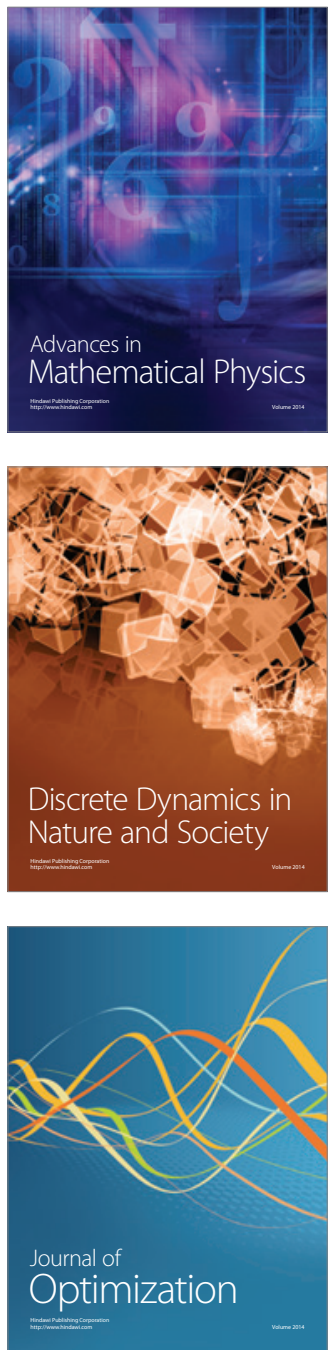